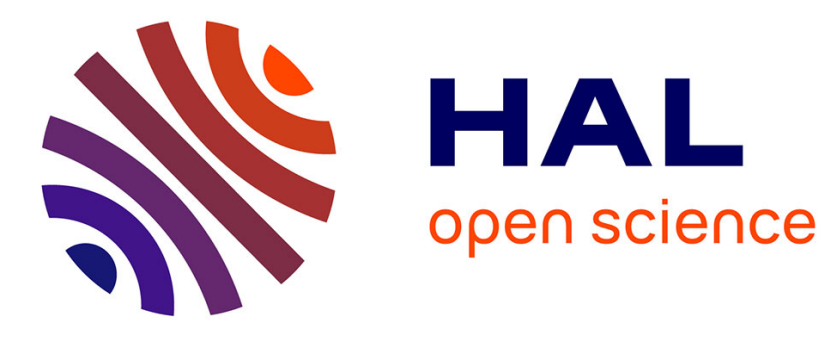

\title{
Les thermes du sanctuaire gallo-romain de Ribemont-sur-Ancre (Somme)
}

Didier Bayard, Jean-Louis Cadoux

\section{To cite this version:}

Didier Bayard, Jean-Louis Cadoux. Les thermes du sanctuaire gallo-romain de Ribemont-sur-Ancre (Somme). Gallia - Fouilles et monuments archéologiques en France métropolitaine, 1982, 40 (1), pp.83-105. 10.3406/galia.1982.1854 . hal-01940299

\section{HAL Id: hal-01940299 \\ https://hal.science/hal-01940299}

Submitted on 6 Feb 2020

HAL is a multi-disciplinary open access archive for the deposit and dissemination of scientific research documents, whether they are published or not. The documents may come from teaching and research institutions in France or abroad, or from public or private research centers.
L'archive ouverte pluridisciplinaire HAL, est destinée au dépôt et à la diffusion de documents scientifiques de niveau recherche, publiés ou non, émanant des établissements d'enseignement et de recherche français ou étrangers, des laboratoires publics ou privés.

\section{(ㅇ)(1) $\$$}

Distributed under a Creative Commons Attribution - NonCommercial - NoDerivatives| 4.0 


\title{
LES THERMES DU SANCTUAIRE GALLO-ROMAIN DE RIBEMONT-SUR-ANCRE (Somme)
}

\author{
par Didier BAYARD ef Jean-Louis CADOUX
}

Le présent article fait suite à une série de trois articles, qui constituaient un premier essai de synthèse sur le sanctuaire gallo-romain de Ribemont-sur-Ancre, et qui ont été publiés en 1978 dans Lalomus ${ }^{1}$. Nous tenterons ici, comme cela a éte fait dans les articles précédents pour le temple, le théâtre et la "zone artisanale " des bâtiments annexes, de résumer et d'interpréter les enseignements de nos deux campagnes de fouilles menées sur les thermes².

Vaste sanctuaire à pèlerinage (fig. 1), le complexe de Ribemont-sur-Ancre fait l'objet de fouilles programmées depuis 1966.

Le précédent article donnait l'état de nos connaissance à l'issue de la campagne de 1975 , alors que les fouilles n'avaient pas encore commencé sur les thermes, qui étaient cependant identifiés, et dont le plan nous était connu, dans ses grandes lignes, par les photographies aériennes ${ }^{3}$. Le sanctuaire a incontestablement des origines préromaines : on les a trouvées sur le site du temple, où un fossé préromain remblayé, sous la partie est de la façade du temple, avait déjà une orientation perpendiculaire à ce qui deviendra l'axe de symétrie principal du sanctuaire. Sous le temple également, des ossements humains dispersés et des armes (époque de La Tène II et surtout La Tène III) attestent la présence d'une nécropole gauloise bouleversée par les constructeurs romains ${ }^{4}$. Il est possible qu'un

1 J.-L. Cadoux, Ln sanctuaire gallo-romain isolé: Ribemont-sur-Ancre, dans Lalomus, XXXVII, 1978, p. 325360. A. Quilli:t, Les enduits peints du sancluaire gallo-romain de Ribemonl-sur-Ancre (Somme), ibid., p. 361-363. F. Drlaci:y, Les sculplures du temple de Ribemont-sur-Ancre, ibid., p. 364-367.

2 Cne publication provisoire a été faite dans le Bulletin trimestriel de la Société des Antiquaires de Picardie : J.-L. Cabolx, Les thermes du sanctuaire gallo-romain de Ribemont-sur-dncre. Premiire campagne de fouilles, 1976, dans Bulletin trimestriel de la société des Anliquaires de Picardie, Amiens, 1977, p. 116-144. Voir également les Informations archéologiques, Picardie, dans Gallia, 35, 1977, p. 317-319; et 37, 1979, p. 329-330.

3 Voir en particulier R. AcicuE et B. Bríart, Atlas d'Archóologie Aérienne de Picardie, Amiens, 1975, p. 110 111 ; mise à jour (voir supra, fig. I) dans R. Acacne, Nouveaux apports des prospeclions aériennes en archéologie préromaine el romaine de la Picardie, dans Cahiers Archéologiques de Picardie, no 6, 1979, p. 60-63.

4 Nécropole ordinaire, ou dépòt sacrificiel comprenant également des sacrifices humains ? Les récentes découvertes de Gournay-sur-Aronde (Oise; 'voir Gallia, Informalions archéologiques, 37, 1979, p. 310-313, ouvrent de passionnants aperçus, qui pourraient avoir leur parallèle à Ribemont-sur-Ancre. 


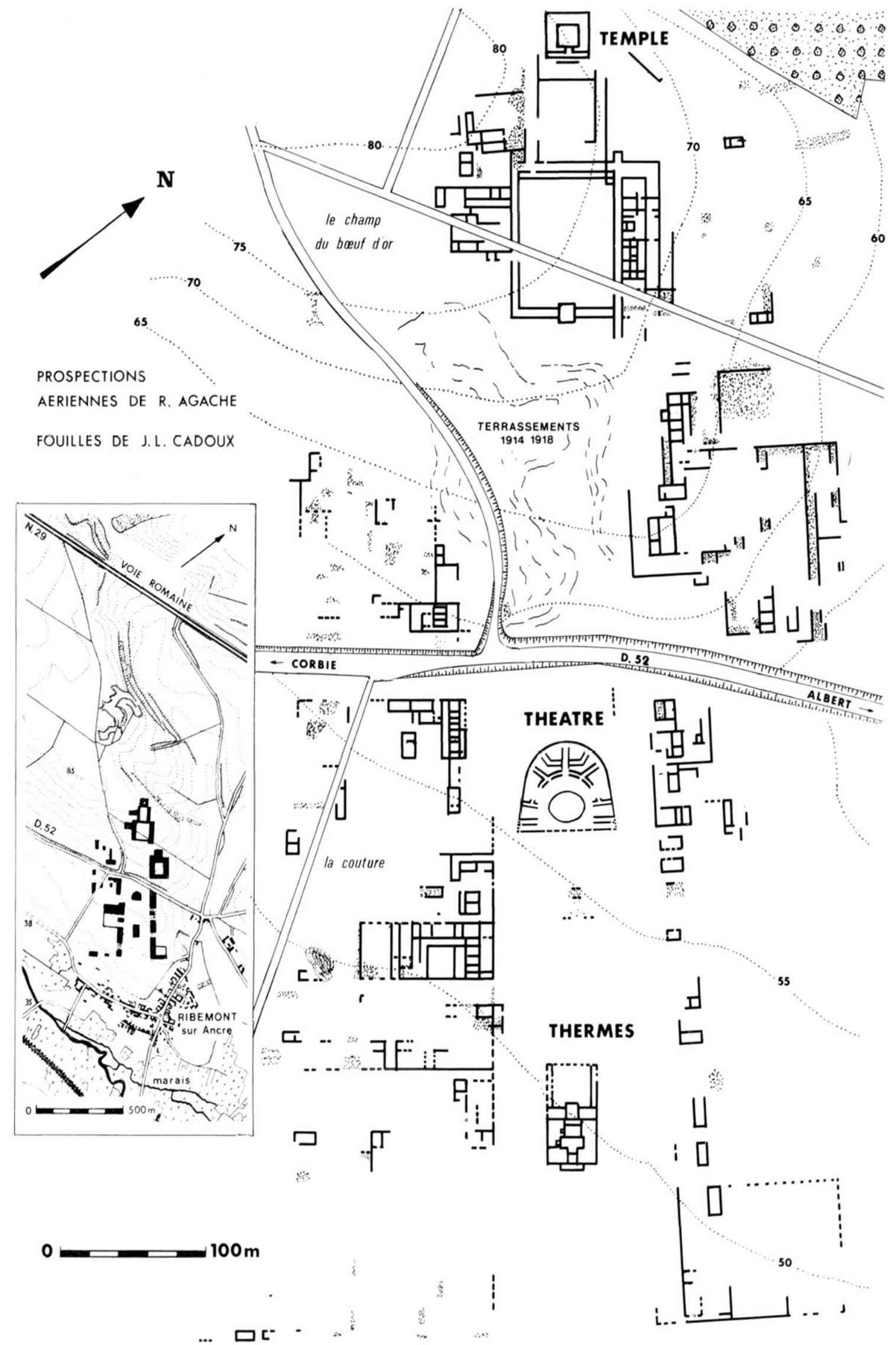

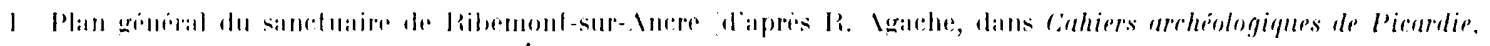

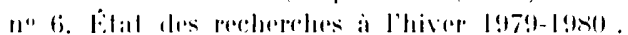




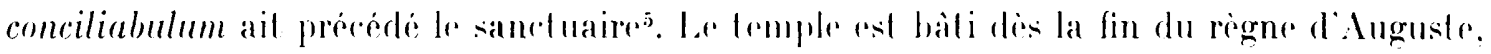
ou sous Tibère, aved d'embléson plan définitil. Le thèatre, dans son premier état, remonte au règne de .éron ou au début du rìgne de Vespasien au plus Lard. Les bàtiments annexes, trop arasés, nont pas pu ètre datés rigoureusement; nous n'avons pas encore pu déterminer non plus si l'orientation secondaire, suivie par les battiments annexes à l'est du sanctuaire, est antérieure ou postérieure a l'axe de symétrie principal. I) moins, une chose est cert aine : immense, concu en fonction d'un axe de symétrie rigide, le sanctuaire a quelque chose d'artificiel. Le temple simble n'avoir eu quiun médiocre succès populaire: pas d'ex-roto, sauf quelques rares fragments épigraphiques au demeurant très soignés. Mieux encore, le temple est détruit penclant le dernier tiers du ier s, et remplacé par un curieux sanctuaire hypetre qui a toutes les apparences d'un sacellum populaire; c'est seulement dans la seconde moitié du $\mathrm{II}^{\mathrm{e}}$ s. que le temple sera reconstruit, luxueusement, avec un riche décor sculpté, pour ne pas connaitre, semble-t-il, plus de succès qu'arant.

Le théatre (fig. 3) au contraire, avec sa capacité d'environ 3000 places, semble avoir fonctionné à plein rendement; dans la seconde moitiés du $\mathrm{II}^{\mathrm{e}}$ s., il est morlifié et devient ensuite un amphithéitre-théatre, encore fréquenté surtout sous Constantin, et sans doute jusqu'au milieu du rves. Pourtant, même si le théătre a un réel succès, le sanctuaire en général semble avoir des difficultés économiques : c'est en ce sens que nous avons proposé d'interpréter l'installation, dès la fin du $\mathrm{r}^{\mathrm{er}} \mathrm{s}$, dans les bàtiments annexes est, d'une zone artisanale qui aurait apporté une activité d'appoint jusqu’à l'abandon du site à la fin du ives.

Le probleme ètait done de savoir si les thermes, avaient eu ou non du sucès ; s'ils avaient eu un succès durable; s ils avaient été réalisés, comme le temple, avec une certaine profusion dans le luxe, ou au contraire, comme le théâtre, avec un souci dominant d'utilitarisme un peu rustique. Il était également intéressant d'étudier dans le détail le plan et les techniques de construction de ces thermes en apparence très simples, presque schématiques, que l'on croirait sortis d'un cahier d'exercices pour élèves-architectes. Il aura suffi de deux campagnes (1976 et 1977) pour mener à bien l'étude des thermes. La surface au sol du monument, des cours (palestre comprise) et de ses abords immédiats, est d'un peru plus de $3000 \mathrm{~m}^{2}$. Les fouilles ont comporlé 29 sondages, pour une surfare totale de $280 \mathrm{~m}^{2}$, soit moins d'un dixième de l'ensemble?

\section{IE PLAN DES THERMES}

A lui seul, l'examen du plan des thermes (fig. :2). tel qu'il découle des photographies aériennes précisées par les fouilles, est très instructif. On olservera d'abord que les thermes sont rigoureusement à cheval sur l'axe de symetrie principal, qui, issu du temple, traverse le théatre en son milieu, pour aboutir aux thermes, situés en contrebas au plus près de la

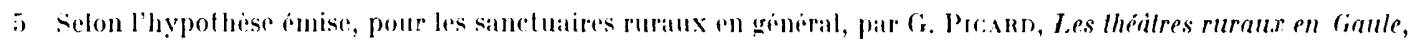
dans Rerue archéologique, Paris, 1970 , p. 1 $1 \times 1-192$.

i Peut-itre, mais pas necessairement, ell méme lemps que le lemple est reconstruil.

7 Il est rai qu il s'agit du dixieme le plus significatif. Toutefois, meme dans les parties foullees d'ume maniere assez exhaustive comme les prefurnia , au moins les banquettes ont ati laisses inlactes. 
rivière ; les thermes sont à sensiblement $600 \mathrm{~m}$ au sud-est du temple ${ }^{8}$; la différence d'altjtude est de $25 \mathrm{~m}^{9}$.

Une enceinte rectangulaire de $58.75 \mathrm{~m}$ sur $33.50 \mathrm{~m}^{10}$ délimite les thermes; la seule structure extérieure à ce rectangle est, au s. ${ }^{11}$, une grosse fondation en " $\mathrm{C}$ " qui s'appuie contre le mur d'enceinte, vraisemblablement un praefurnium. A l'intéricur du rectangle, deux parties se distinguent : au n., la palestre, bordée intérieurement de portiques à l'e. et à l'o. ; avec une longueur, dans le sens de l'axe de symétrie, de $28,15 \mathrm{~m}$ environ, la palestre occupe un peu moins de la moitié de l'enceinte. Tout le reste est dominé par le bâtiment des bains où se succèdent, du n. au s., les trois pièces classiques : le frigidarium, dont la façarle, au n.. mord sur la palestre; le lepidarium, au centre : il est subdivisé en deux pièces, la plus petite, au n., étant peut-être l'apodylerium. Il est flanqué, à l'o., d'un praefurnium carré. Enfin, le caldarium est la pièce la plus grande, avec à l'o. un grand praefurnium, et au s. une longue abside, qui lui donne un plan en "T ", et où il faut peut-être reconnaître le laconicum ou le sudalorium. Le reste de l'enceinte est occupé par deux courettes de part et d'autre du frigidarium, et les cours de service de part et d'autre des deux pièces chauffées.

On trouve donc à Ribemont-sur-Ancre, traitéc avec une élégante simplicité, la "disposition centrée et symétrique $\|^{12}$ où se succèdent, sur l'axe d'un rectangle, à partir de la façade, le frigidarium, le lepidarium et le caldarium, tandis que se balancent, de part et d'autre, les locaux de service. Postérieur à l'"ordonnance excentrique» et au "dispositif centré asymétrique ${ }^{13}$, ce lype de plan, qui va triompher à partir du III ${ }^{\mathrm{e}}$ s, se rencontre en particulier dans les grands thermes du Nord à Timgad, et connaît son apogée dans les thermes de Caracalla à Rome, puis ceux de Sainte-Barbe à Trèves. Cette disposition centrée et symétrique, la plus tardive donc, apparait à Ribemont comme sur une épure, d'autant plus que le plan, comme on peut l'observer, ne comporte aucune courbe, ce qui est fort rare dans les établissements de bains. On retrouve quelque chose de comparable dans les "thermes du forum ", à Saint-Bertrand-de-Comminges ${ }^{14}$.

La comparaison avec les thermes du sanctuaire de Champlieu (commune d'Orrouy, dans l'Oise) est tout aussi frappante ${ }^{15}$. Les thermes doubles du sanctuaire à pèlerinage (?)

8 On compte $625 \mathrm{~m}$ du centre de la cella du lemple au frigidarium des thermes.

9 La cella du temple est sur la courbe de niveau des $75 \mathrm{~m}$ (niveau acluel du sol ; les thermes sont sur la courbe des $50 \mathrm{~m}$. La pente devient beaucoup plus faible à partir du mur de scene du théatre, ou le niveau actuel du sol ne depasse pas $57 \mathrm{~m}$, à un peu plus de $250 \mathrm{~m}$ au nord-ouest des thermes.

10 Mesures exterieures. En prenant pour base un pied romain post-flavien d'environ 0,295 m (voir 11 . Novotxy, Metrologische Nova, dans Klio, 24, 1934, p. 257 et s.), on aurail une longueur tries proche de 200 pieds et une largeur de 114 pieds, à moins d'un pied pres.

11 Par convention, el pour éviter des lourdeurs inutiles, nous appellerons " còté sud " le petit còté de l'enceinte regardant la rivière, bien qu’il soit en réalité orienté vers le sud-est; "coté nord "le pelit còté qui regarde vers le théatre; etc.

12 F. Bexoît, dans Daremberg-Sabljo-Pottikr, I)iclionnaire des Anliquilés ..., s.v. thermae, V, p.218-219.

13 Ibid.

14 Voir A. Grwive, Manuel d'Archéologie gallo-romaine, IV. Paris, 1960, p. 279-283.

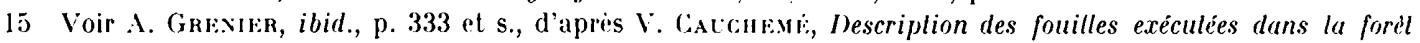
de Compiègne sous la direction de M. A. de Roucy :et non "de Roncy", coquille d'A. Grixirr, Ibid., p. 33.4, note 1 , IV, Compiegne, 1912 . 
d'Allonnes, près du Mans, ont un plan très proche de ceux de Ribemont ${ }^{16}$. Les thermes de Jublains-Noiodunum (Mayenne), partiellement reconnus en 1876, viennent de faire l'objet d'une fouille exhaustive'17. Leur plan présente une claire parenté avec ceux des thermes de Xanten-Colonia Llpia Trajana, en (iermanie Inférieure, ceux du vicus de Heerlen-Coriovallum, dans les Pays-Bas actuels, et ceux de Cologne-Colonia Claudia Ara Agrippinensium ${ }^{18}$. Dans ces trois exemples, ce n'est pas encore le plan "centré et symétrique», comme à Ribemont et Champlieu, mais on s'en approche beaucoup. L'architecte y reste en effet attaché à la tradition du "dispositif centré asymétrique». A une époque un peu plus tardive que celle de la construction des thermes de Ribemont, un plan "centré et symétrique " très voisin de celui des thermes de Champlieu, semble l'emporter dans les camps du limes rhénan: ainsi à Niederbieher ${ }^{19}$, à la Saalburg ${ }^{20}$, à Rottweil-Arae Flaviae ${ }^{21}$. C'est surtout à partir du règne d'Hadrien que ce type de plan semble se généraliser ${ }^{22}$ y compris dans des thermes de villae comme à Nennig. Quelques comparaisons encore plus instructives à bien des égards : le plan des thermes centraux d'Augst-Augusla Raurica (Suisse), insula XXXII, est de la mème veine que celui des thermes de Ribemont. rigoureusement axé, sans une seule courbe, avec un caldarium en croix : la principale différence est que, en plus des trois pièces canoniques, les thermes d'Augst comportent des pièces complémentaires qui ne figurent pas à Ribemont ${ }^{23}$. Or nous avions autrefois remarqué que le plan du théâtre de Ribemont n'est pas sans parentés avec celui des théâtres superposés d'Augst : disposition des vomiloria dans le premier théâtre d'Augst (augustéen) et dans le premier étal du théatre de Ribemont (vers 60) ap. J.-(i. ?) ; recours systématique aux contreforts en arc de cercle à Ribemont dès le premier ètat, à Augst dans le second état (vers 150) ap. J.-(i. $)^{24}$. Il est donc possible que, à plusieurs reprises, les architectes se soient servis des mêmes modèles, pour des réalisations plus élaborées à Augst, plus modestes et simplifiées à Ribemont. Non loin d'Augst, à Avenches, dès l'époque flavienne, les thermes "En Pevinet" ont encore un plan du mème type ${ }^{25}$.

Ce plan des thermes de Ribemont-sur-Ancre, si caractéristique, presque schématique,

16 Voir A. Grixis, Manuel .... IV, I, p. 323-326. Plus recemment, J. Barie el collab. Allonnes dansl'.1nliquilé, Faculte des Lettres du Centre Lniversitaire du Mans, le Mans, 1974.

17 Voir G. Alsix, Informations archéologiques Pays de la Loire, dans Gallia, 38, 1980, p. 382-384.

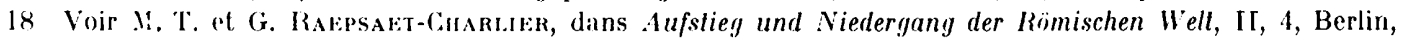
1975, p. 132-137. our Cologne, voir H. Hıx, ibid., p. 844.

19 Voir (Grexiler, op. cil., IV, p. $3 \times 2$.

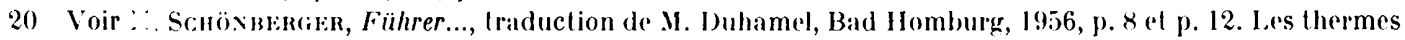
sont donnes comate confemporains du grand camp pour cohorle, construits sous le regne d'Iadrien. Voir igalement Cih. II. Trkxws, dans Aufstie! und Niedergang..., II, 5, 2, Berlin, 1976, p. 810-811.

21 Voir A. R̈̈scu, dans Aufstieg und Viedergang..., rr, 5, 1, Berlin, 1976, p. 587-5x8. Il s'agil du Bain 3, silue: a l'inférieur du camp II h, et donne comme "spättaianischer" ou plus vraisemblablement "hadrianischer".

2: On pourrat ajouler a ces exemples ceux de Tindonissa ef de lleddernheim, ef celui, plus recemment decouverl, de Walldürn en Krifie, voir D). Plaxk, dans .1ufstieg und Tiedergang ..., I1. 5, Berlin, 1976, p. 424-425; etc. Pour les thermes de villae, le plan axial apparait des le début du ne s., a la villa de Fontorle a Cadrien (l,ol, mais peut-etre imposé par le remploi d'une enfilade de pieces plus anciennes. Voir W. Labrocsse, Informations archéologiques, Midi-Pyrénées, dans ciallia, XXVIII, 1970, p. 423.

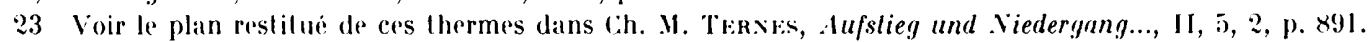

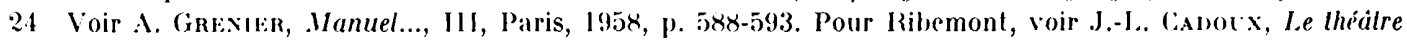
gallo-romain de Ribemonl-sur-Ancre: essai de synlhese, dans Cahiers archénlogiques de Picardie, II, 2, 1975, p. 29-54.

25 Voir cih. M. Trkxks, op. cil., p. 91:2-913. 
fait encore figure de rarete a la date, tries précoce, bers 100 ap. J.-ti., oi lédifice est construit. Si nous prenons le cas de la ville de Timgarl aver un terminus posl quem parfaitement connu nous constatons que sur 13 établissements de hains idlentifiés, un seul, les "grands thermes du Nord", possède le plan "centré et symetrique "26. Jes thermes de Ribemont se situent donc tres tot dans un ensemble où l'on voil les architectes romains, partant du schéma "excentrique " primilif, s'acheminer vers le schéma "centré al symétrique "qui s"imposera à Rome mème avec les hermes de Cararalla, et triomphera jusquau IV s. avec la grande série des thermes impériaux ${ }^{27}$. Quelles peuvent être les raisons qui ont conduit l'architecte des thermes de Ribemont a adopter, a une date si haute. un plan si moderne? On ne peut qu'emettre des hypotheses. Tout d'abord, la place ne manquait. pas, aucune contrainte née de l'environnement ou de constructions antérieures ne venait gêner l'architecte. Ies conditions étant propices, il n'est pas invraisemblable de penser qu'on avait moins de scrupules a installer che\% les paysans Ambiens des thermes d'une conception encore nouvelle, lont les applications étaient encore trop rares et trop récentes, et qu'on avait encore à expérimenter ${ }^{28}$. Le choix d'un lel lype de plan, surtout sous l'aspect rudimentaire retenu pour Ribemont. a dì aussi bien etre dicté par un souci d'économie: une construction de ce genre était certainement moins coùteuse. Mais il est probable que les frais de fonctionnement étaient plus élevés : nous reviendrons plus loin sur cette irlée; c'est en effet peut-itre une des raisons pour leduelles ces thermes ont éte abandonnes at démontés après, approximativement, un siècle d'utilisation sinulement.

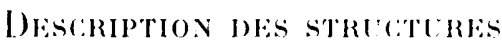

Les thermes du sanctuaire gallo-romain ont ete retrouves, dans la limite des sondages effectués, très profondément arasés : pas une seule pilette dhypocauste n’útait restée en place, et parfois les murs, entièrement récupérés. nont pu itre reconnus que par le hourrelet de mortier laissé sur la fondation de craie le long de la première assise de moellons. Malgré cela, et au prix d'une fouille extrèmement méticuleuse, nous avons pu obtenir de nombreux renseignements; notre tâche a été, il est vrai, facilitée par le fait que la récupération des thermes. survenue des l'Antiquité, arait été systématique ot orronnée, ol qu'elle n'avait été suivie d'aucune perlurbation ultérieurese.

26 Les "grands thermes du Xord" a Timgad, ont ite bien chludies at bien publies pour leurs struclures.

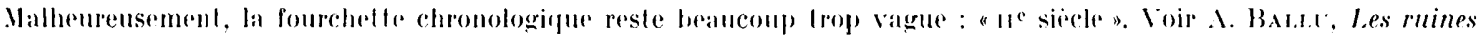

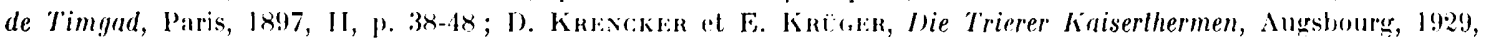
1. 231-233; at ricemment: S. German, Les mosü̈ques de Timgad, C..X.R.S., Paris, 1969, p. 71-73.

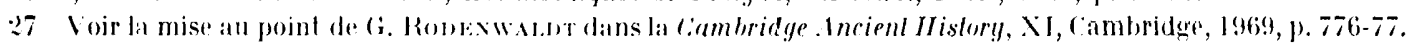

ix On observe un phenomene comparable ave certains forn de province : Jes fora d'Alesia at de silchester Calleva, dont te plan semble s'inspirer de celui du forum de Trajan, a Rome, lui sont en rialite anterieurs. Voir

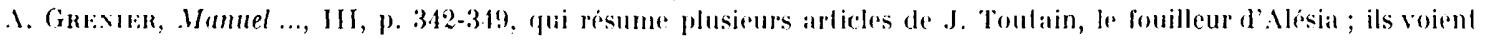

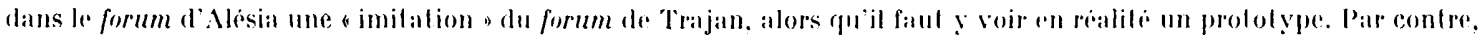

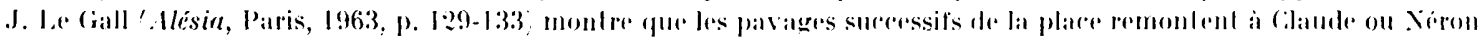

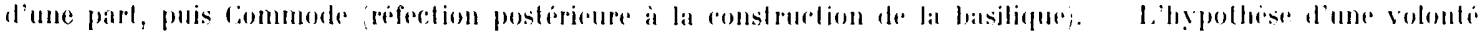

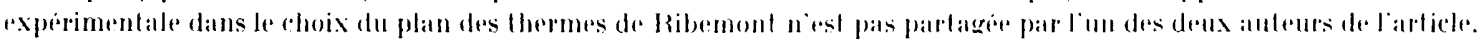

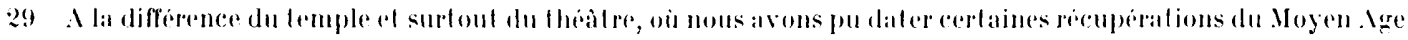

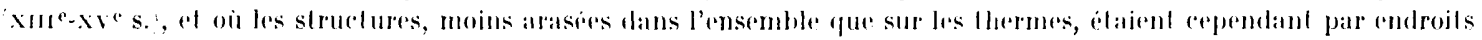
completement boulererseres. 

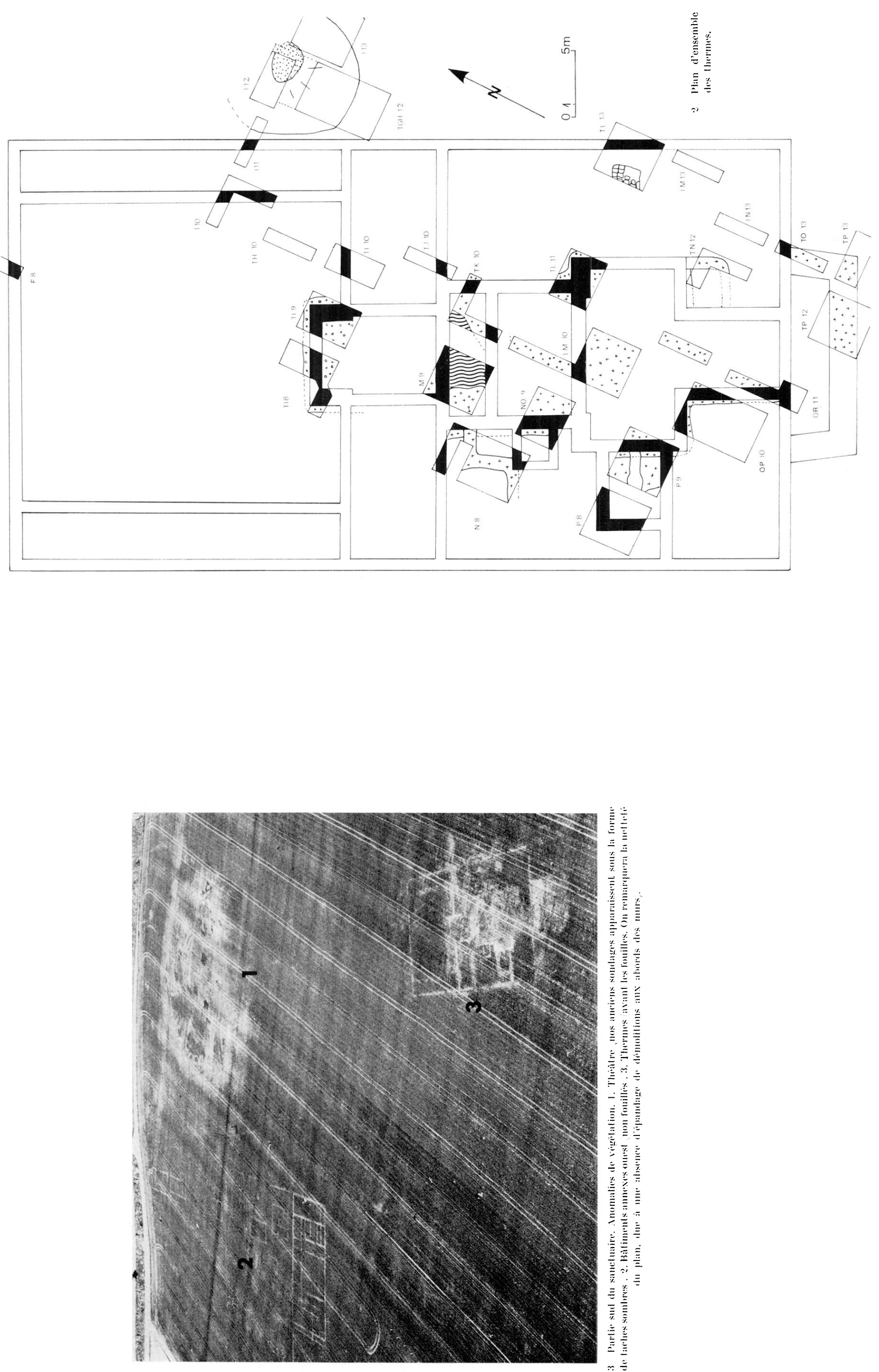


\section{Le mur exlerieur et la palestre.}

Les thermes sont inserits dans un grand rectangle de 58.75 m sur :3,3,in m. ferme par un mur d'enceinle. Comme a Champlieu. toute la parlie n. des thermes est orcupée par une grande cour. presque carrée (2:3.6) $n$ sur $2.2 .70 \mathrm{~m}$. mesures intérieures, non compris les portiques latéraux ou lon reconnait la palestre. Ia partie s. est oceupee par le bitimenl des bains, avec, de part et d'autre, des cours de servire. seule deborde de cette enceinte une grande fondation en "l" " au s. des llermes. vaisemblablement celle doun praefurnium. Le mur denceinte a ele mis au jour dans fi sondages:

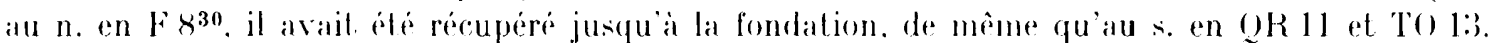
(In a pu observer que la fondation. en raie lassee, avait une largeur de $0,80 \mathrm{~m}$ a 0.90 m. et une profondeur d'un peu plus de $10.60 \mathrm{~m}$. A l'e. TT. 1:3, TG-H 12 et 111 , il subsistait du mur d'enceinle deux a trois assises de moellons : on a pu constater qüil élail régulierement paremente de petils

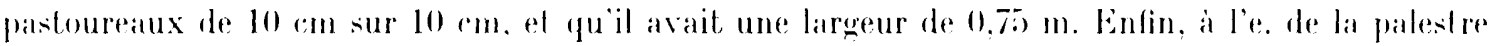
sondages To 12. I 12 et I 13). le parement extérieur du mur d'enceinte avait bascule d'un seul tenant sur la grande fosse dont il sera question plus loin : on a en effet relrouve. srellant la partie o. de la fosse, un lit horizontal de moellons, la face en bas et la pointe en l'air, jusqu’a une distance de 4 m exaclement du mur. Le mur d'enceinte avail done une hauleur minimale de 4 m.

Quelques sondages limites faits a löntérieur de la palestre (TII 10 surtout n'ont pas permis de reconnaitre le sol antique. I es stratigraphies montrent du moins une différence de couleur assez tranchée entre l'argile jaune inférieure (loss en place) et l'aruile grrise remaniée au-dessus. I)ans less quatre sondages. le niveau supérieur de l'argile grise est sensiblement horizontal, et les démolitions ipandues a partir des murs, sont posies directement sur lui avec quelques plarages d'arqile jaune. Il est done tries vaisemblable que le sol de la palestre était fait de simple terre baltue.

bin bordure de la palestre. a l'e et a l'o.. se trouvait un portique. dont la foulle (sondage I 10) n'a permis de retrouver que la fondation : une fondation de craie continue large de 1 m. profonde d'au moins (0.5) m. Il ne subsistait ni assise de moellons, ni trace d'implantalion de bases de colonnes. la largeur du portique est de $3,20 \mathrm{~m}$ (mesures intérieures. Les photographies aériennes fonl apparaitre des traces de ce qui pourrait aussi avoir été un portique au n. de la palestre, à l'extérieur du mur d'enceinte. coté théatre. Des anomalies de végétalion, visibles surtout dans les angles no-o. el n.-e., devant le mur d'enceinte, donnent l'impression d'une fondalion discontinue, qui aurail pu supporter des rolonnes distantes d'a peu pres :3 m les unes des autres. Mars la seule structure vue en fouilles (sondage F 8 i est un amas de raie lassée a peu pres informe, peu épais (0,40 m, contre 0.66 m d'epaisseur pour la fondation du mur d'enceinte au s. du mème sondagej, bloigné de 2.30 m seulement du mur d'enceinte. Ces lambeaux peuvent-ils correspondre aux fondations d'un portipue monumental? Pourquoi re portique serait-il, à la différence des aulres. extérieur au mur d'enceinle? Bornons-nous à constaler que, vu l'état d'arasement des vestiges, le problime de l'aspect d'une éventuelle entrée des thermes colfe theatre, n'est pas résolu.

On aimerait aussi pouvoir identifier les deux courettes?? silués de fart et daul re du frigidarium. seule la couretle e a élé sondée. TI 10 et T.J 10). Jille a une largeur de 6,30 m et une longueur de $11.80 \mathrm{~m}$. Le mur colé palestre. dont il restait :3 assises de moellons parementes de 10 cm sur 10 ('m. a 0.75 m d'epaisseur. l.e mur s. elail entieremenl récupéré ; il nen subsistait que la fondalion

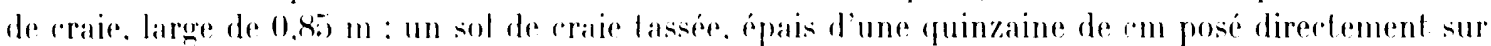

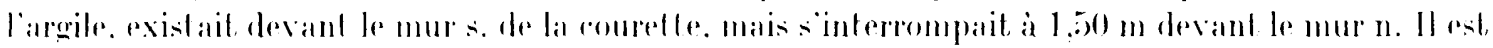

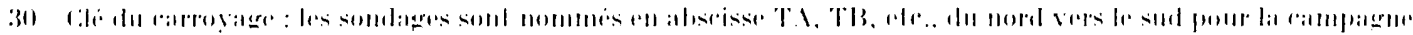

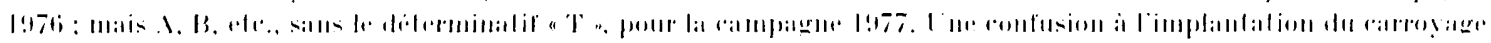

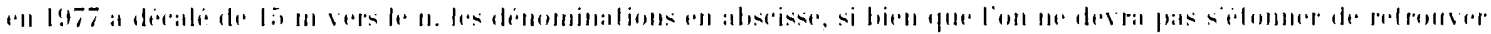

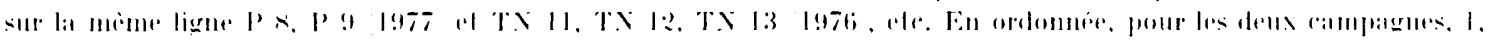

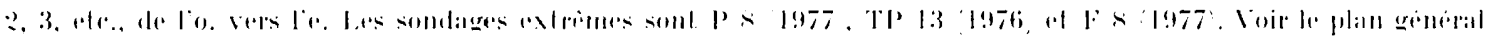
des llirmes, fige. 3. 
difficile, partant de ces maigres éléments, de dire s'il s'agit de pièces couvertes (double apodyterium? prolongation des portiques de la palestre?) ou de simples courettes à ciel ouvert (courettes de service avec un sol rudimentaire en craie damée?).

\section{Le bâlimenl des bains.}

Ce qui frappe, comme on l'a vu, dans la disposition du bâtiment des bains, c'est sa simplicité presque schématique : alignées selon un plan centré le long de l'axe de symétrie général du sanctuaire, se succèdent du n. vers le s., les trois pièces canoniques, frigidarium, lepidarium et caldarium, avec un élargissement progressif de chaque salle. Le frigidarium a en effet environ $5,15 \mathrm{~m}$ de large (mesures intérieures) dans sa partie n., débordant sur la palestre, puis 5,75 m dans sa partie s.; le lepidarium a $9,30 \mathrm{~m}$ de large ; le caldarium atteint $13 \mathrm{~m}$ dans sa partie n., pour retrouver, dans l'abside au s., les $5,15 \mathrm{~m}$ de large de la partie $\mathrm{n}$. du frigidarium ${ }^{31}$.

Une autre originalité des bains est que les trois pièces reposent sur un radier continu (un "socle»? un "soubassement"? on ne sait comment nommer cette structure à peu près unique en son grenre) en craie tassée, très dure, qui sert de fondation à la fois pour les murs, les hypocaustes et les sols des pièces non chauffées. L'ensemble des bains repose sur ce radier un peu à la manière des maisons contemporaines qui, en terrain instable, reposent sur une dalle de béton.

On comprend aisément la méthode utilisée par les constructeurs gallo-romains pour faire ce radier, uniformément épais de $0,60 \mathrm{~m}$ : ils ont dû commencer par tracer le plan au sol des bains, puis creuser dans l'argile sur toute la surface du bâtiment; ils ont ensuite déversé la craie en mouillant abondamment et en tassant au fur et a mesure. L'opération a dû être faite en deux fois au moins, car on voit en plusieurs endroits, là où les coupes ont été effectuées, la trace des reprises, et même un niveau d'argile (rebord n. du radier dans le sondage TI, 11) épandu dans la craie à $10 \mathrm{~cm}$ environ sous le haut du radier. Le radier n'est pas rigoureusement plan : il suit la pente douce du terrain, descendant du $\mathrm{n}$. vers le s. avec une différence de niveau de $0,64 \mathrm{~m}$ entre le frigidarium (sondage TI 8) et l'abside du caldarium (sondage T.X 11). Ce parti pris, outre qu'il était le plus économique, avait l'avantage de faciliter les transitions entre le niveau, moins élevé, des sols des salles non chauffées, au n., el celui des salles sur hypocauste, au s. Le radier avait aussi un léger pendage de l'e. vers l'o. (une dizaine de centimètres à la hauteur du caldarium) : les constructeurs n'ont pas cherché à le compenser, et se sont contentés de rattraper la différence de niveau au moment de la construction des murs ; ainsi, l'assise inférieure de moellons du mur n. du caldarium (sondage TM 10) est taillée en biseau, avec des pastoureaux de moins en moins hauts de l'o. vers l'e. Quelques négligences enfin ont été observées : le radier est beaucoup moins tassé sur les bords, où il forme un bourrelet. Il déborde en moyenne de $0,40 \mathrm{~m}$ à l'extérieur des murs (sondages TI 8 , TI 9, QP 10), mais devant l'angle n.-e. du caldarium (sondage TL 11), il coupe court et passe juste sous le pied du mur, qui de la sorte repose pratiquement en surplomb sur le bord d'une fondation qui n'est même pas verticale.

Quoi qu'il en soit, ce radier, très dur et étanche, devait constituer, en même temps qu'une fondation, un excellent isolant thermique. C'est une technique qui, a notre connaissance, ne se rencontre nulle part ailleurs dans le monde romain, sauf un autre exemple assez mal connu à Samarobriva : rue Jeanne-Xatière, au moment de la reconstruction d'Amiens. F. Vasselle a observé une pièce de $14 \mathrm{~m}$ de large, traversée par un égout, et dont la moitié $\mathrm{n}$. semble avoir été chauffée par hypocauste. Les murs, les sols de béton rose et l'égout reposaient sur "un radier de craie tassée d'une épaisseur de $1,80 \mathrm{~m}$ », creusé dans le sol vierge, ici du gravier ${ }^{32}$. C'est bien la même technique qu'à

31 Comple tenu d'inévitables imprécisions 'de l'ordre de $5 \mathrm{~cm}$ pour charque mesure), ces dimensions pourraient se traduire en pieds romains de $0,2957 \mathrm{~m}$, respectivement par : 18 p., 10 p., 32 p., 45 p. et à nouveau 18 p.

32 Voir F. Vassili.t.: dans Bulletin de la Société des Anliquaires de Picardie, Amiens, 1952, p. 158-160. La mème technigue se relrouve a Ribemont mème dans les bâtiments annexes E. en cours de fouille. Il semble s'agir ici d'hypocaustes chauffant des pièces à l'extrémitó s. d'un habitat assez luxueux non encore daté. 
Ribemont-sur-Ancre, mais la construction est ici plus massive et plus tardive, au plus tòt la deuxième moitié du $\mathrm{II}^{\mathrm{e}} \mathrm{s}^{3}{ }^{33}$.

Le frigidarium est une simple pièce rectangulaire dont la grande longueur, dans le sens n.-s., est de $8,40 \mathrm{~m}$ (mesures intérieures) ${ }^{34}$. L'épaississement intérieur du mur $\mathrm{n}$, dans la partie qui déborde sur la palestre, complique cependant le plan, en donnant à cette partie du frigidarium l'aspect d'une sorte de petite abside (fig. 4). En ce qui concerne le mur n., son angle o. avait été entièrement récupéré, et il n'a pu être localisé que par les traces de mortier qu'il a laissées sur le radier de craie. La présence d'un bourrelet continu de mortier au pied de la première assise des moellons disparus a permis du moins de situer le mur avec une précision parfaite. L'angle n. par contre restait dans un bon état de conservation, sur 8 et 9 assises de moellons (sondages TI 8 et TI 9 ).

Le mur de façade du frigidarium, còté palestre, a $0,95 \mathrm{~m}$ de large. Il est impossible de dire s'il y avait une entrée sur la palestre, l'arasement ayant été poussé au-dessous du niveau d'un seuil éventuel. Toutefois, en cet endroit, une entrée est peu vraisemblable : elle aurait donné directement sur la piscine qui occupe habituellement l'abside des frigidaria. Les murs latéraux de l'abside sur la palestre ont $1,16 \mathrm{~m}$ de large, soil 1 pied de plus que le précédent. Le mur s. (sondage. $\mathrm{M}$ 9). conservé jusqu'à 9 assises de moellons, a une largeur de $0,87 \mathrm{~m}$, soit 3 pieds. Il a été récupéré dans l'angles. du frigidurium ; plus à l'e., là où le mur sépare le frigidarium de la pièce n. du lepidarium, l'arasement s'est arrèté à un niveau de mortier parfaitement plan au-dessus de la 9e assise de moellons, à un peu moins de $10 \mathrm{~cm}$ plus haut que le sol en place de la pièce n. du tepidarium : n'y aurait-il pas eu un simple emmarchement entre les 2 pièces?

A l'intérieur du frigidarium, le sol n'était plus en place. Si son niveau antique nous échappe, on peut du moins reconstituer l'aspect de ce sol, qui a été bouleversé, mais non récupéré. Un hérisson de gros blocs de craie était posé sur le radier de craie tassée ; il supportait un sol de béton rose très épais (environ $20 \mathrm{~cm}$ ), dont plusieurs gros morceaux basculés ont été retrouvés. Leur face supérieure portait en négatif (bourrelets dans le béton) la trace de legulae disposées en quinconce. Il est vraisemblable, mais pas certain, que ces tegulae servaient de base à des dalles en calcaire fin dont de nombreux fragments ont été trouvés dans les démolitions.

Le tepidarium: il y a en fait deux pièces, et la pièce n. est peut-être à rattacher comme on l'a vu, au frigidarium. Si l'on prend le plan de masse, on constate un premier élargissement, et l'on arrive à une grande pièce double sensiblement carrée de $9,30 \mathrm{~m}$ sur $9 \mathrm{~m}$, bordée au n., à l'o. et à l'e. par des murs de 3 pieds, séparée au s. du caldarium par un mur large d'un peu plus de $1,15 \mathrm{~m}$. Cette pièce est subdivisée en deux par un mur large de $0,75 \mathrm{~m}$. La partie n. est une sorte de couloir large de $2,70 \mathrm{~m}$, non chauffé. La partie s. seule était sur hypocauste ${ }^{35}$.

La pièce n. conservait, partiellement en place, son sol, une sorte de terrazzo en béton rose avec inclusions de silex noirs, de morceaux de brique rouge et de morceaux de craie, assez décoratif, posé sur un hérisson de gros blocs de craie, lui-même posé sur le radier de craie tassée. Ce sol était à peu près certainement en léger contrebas par rapport à celui du frigidarium. Il était en contrebas d'au moins $50 \mathrm{~cm}$ par rapport au sol (disparu) du lepidarium chauffé̉ ${ }^{36}$. Enfin le mur séparant la pièce n., de la pièce s. chauffée, devait avoir une certaine élévation : il en reste en place, au-dessus du sol de la pièce $\mathrm{n}$., une assise de moellons vue au s. des sondages $\mathrm{M} 9$ et TK 10 , avec un blocage bien apparent au-dessus duquel on n'a pas trouvé de niveau de mortier à surface plane, qui serait l'indice d'un seuil ou d'un emmarchement. Ainsi la pièce n., ouverte semble-t-il sur le frigidarium, ètait séparée

33 Ine monnaie d'Hadrien a dete trouvée sous le radier.

34 Soit 29 pieds. Surface couverte, compte tenu du rétrécissement, au n., de la partie débordant sur la palestre: $46,9 \mathrm{~m}^{2}$

35 Surface de la piece $\mathrm{n}$. : $25,1 \mathrm{~m}^{2}$. Surface du lepidarium chauffe : $42,6 \mathrm{~m}^{2}$.

36 Le niveau de hase de l'hypocauste arasé est donné par la bouche du praefurnium dans le sondage No 9 : ce niveau de base est a peine $25 \mathrm{~cm}$ plus bas que le niveau supérieur du sol de lerraza dans la pièce n. 


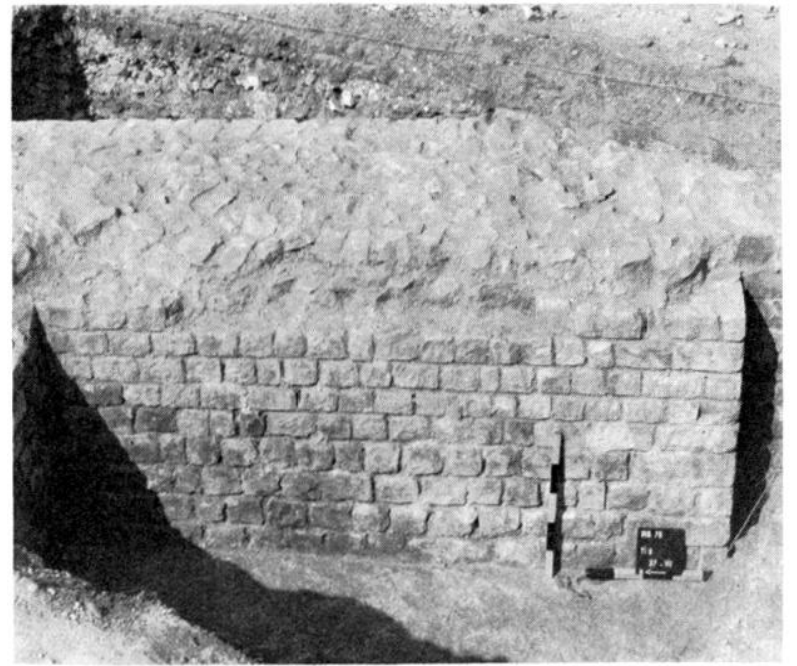

4 l.angle nord-est du frigidarium. (On observe gure le nivean d'arasement est partialement. plan.

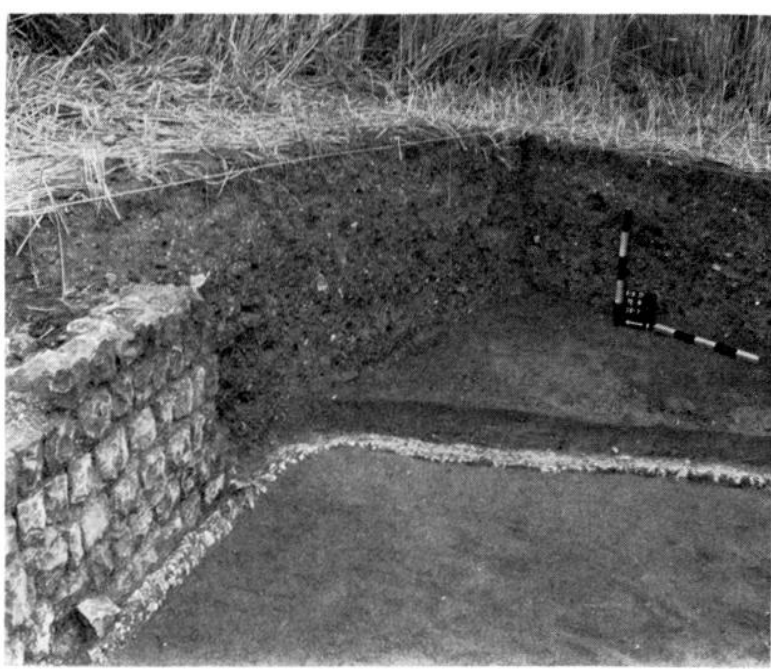

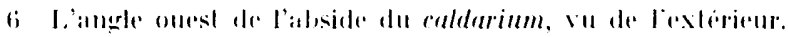
1 yauche. 10 mur sud du crldarium. A droile, la mur de

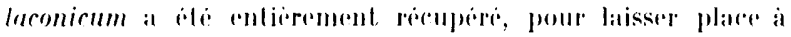

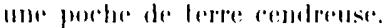

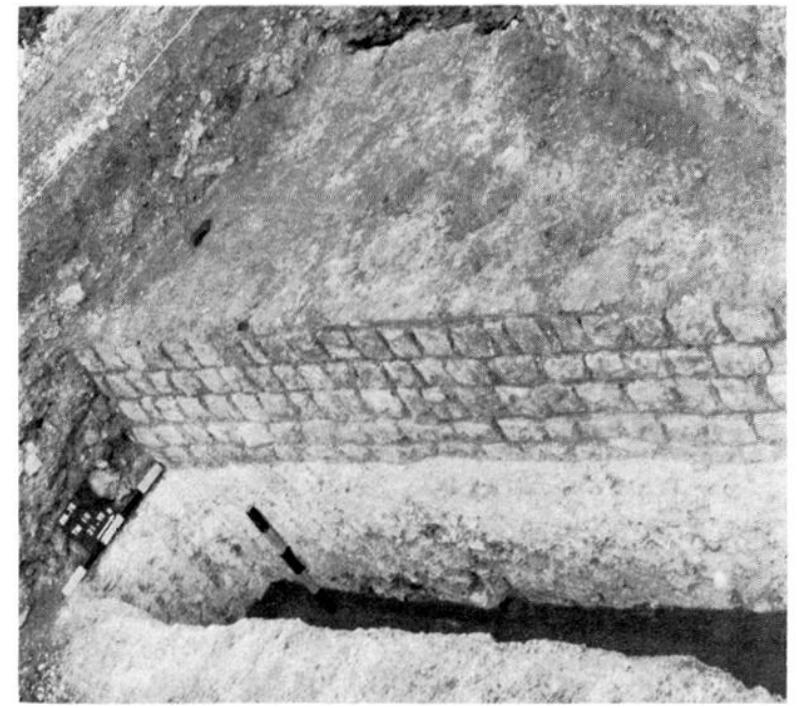

5) I.e mur de siparalion entre te tepidariam et le caldariam, face sud. En haul, niveaul de mortier aver ampreintes de

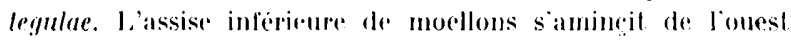
vers l'esi, pour matlaper lhorizontale. Ln bas, coupe a lancers le radier.

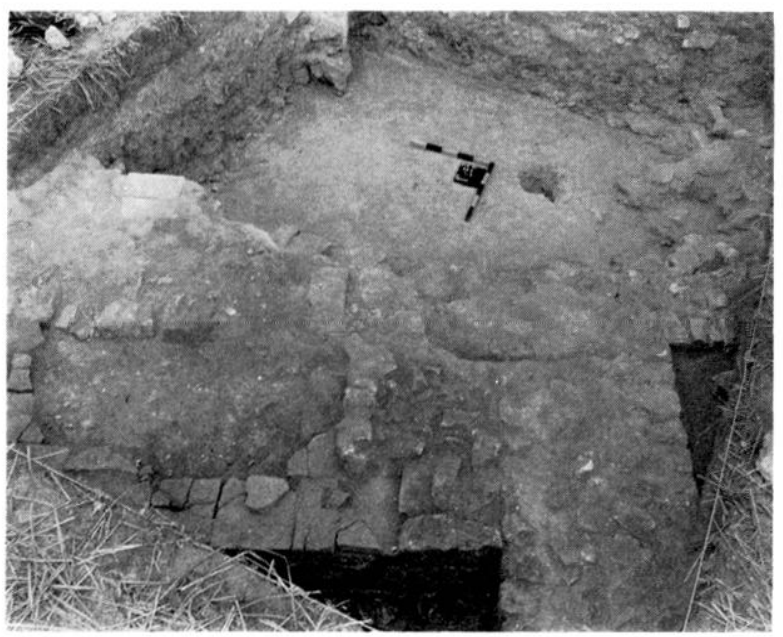

7 Dedail du frrefurnium du tepidurium. An premier plan,

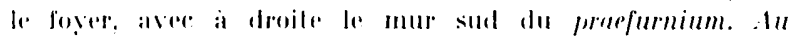

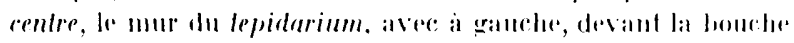

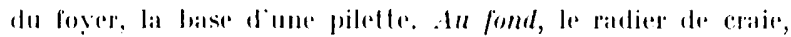

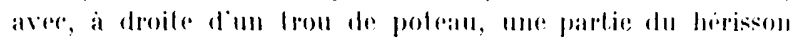

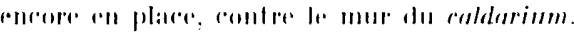

du tepidarium par ce mur, aver peut-itre des portes liatcirales. puisque, comme on vient de te voir, il ne pouvail pios y avoir de porte axialle.

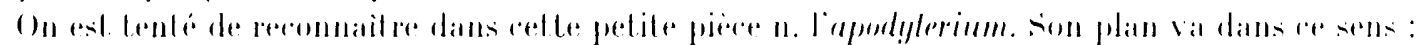

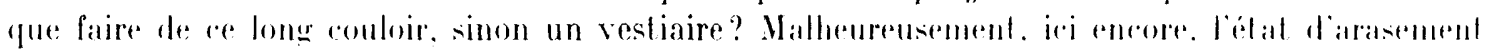

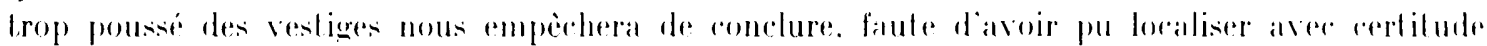
lemplarement des portes. 


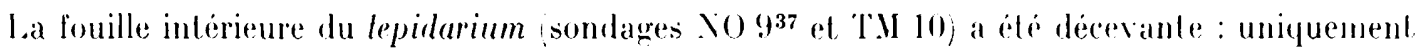
des démolitions posées directement sur le radier de craie. Ia récupération arait été poussée jusqu’a la base de l'hypocauste. Mais les observations faites a la bourhe de son praefurnium (sondage No 9, et sur le mur de séparation entre le tepidarium et le caldarium. nous ont permis de comprendre l'agencement de cet hypocauste. I.e pavage de luiles du fover du prafurnium subsist ait largement en place jusqu’a la bouche du praefurnium au bord intérieur du mur o. du tepidarium. Ce paragere rlail is $0,50 \mathrm{~m}$ au-dessus du radier de craie.

l.e haut du mur de séparalion entre lepidarium el caldarium. lel que nous larons rel rouví. itait à $0,60 \mathrm{~m}$ au-dessus du radier. Il était parfait ement plan. couverl d'une courhe de mort ier rose oin se lisait encore bien en négalif. par une série de rainures dans le mortier, la trace d'un dallage de luiles (larges de $0.20 \mathrm{~m}$ a 0.25$)$ m) proses còte a cote. Ces deux ohservations nous donnent te nivean de base de l'hyporauste, dont il ne reste rien. Comme dans le frigidarium. on avail le radier. puis un hérisson de craie épais de $0.50 \mathrm{~m}$ a $0.60 \mathrm{~m}$. selon les corrections apportées a la pente du radier. puis, non plus ici un sol, mais la base de l'hypocauste constituée d'un pavage de tuiles, el enfin le: pilet.es el la suspensura, dont il ne reste rien.

Ia seconde observation d'autre part, prouve qu'il y avait continuité entre l'hypocauste du lepidarium et l'hypocauste du caldarium - comme c'est le cas aussi bien à Champlieu, etc. Les deux pièces ne pouvaient ètre séparées que par des cloisons légrires ou des tentures (fig. j). Y arait-il une arcade entre les deux pièces, ou une simple poutre horizontale? Peut-être une arrade, re qui expliquerait pourquoi, dans les angles n.-o. et n.-e. du caldarium, le mur de séparat ion entre les deux pieces s'épaissit d'un pied, passant de $1,16 \mathrm{~m}$ a $1,45 \mathrm{~m}$, comme s'il devait jouer le ròle d'un cont refort (sondage TI, 11). Mais une fois de plus, l'arasement des vestiges est trop poussé pour qu'on puisse conclure.

Le caldarium, long de 14,40 m (mesures intérieures) ${ }^{38}$, aree un plan en " $T$ " ". se divise. au 11. ." une grande pièce de $6,50 \mathrm{~m}$ sur $13 \mathrm{~m}$, et au s., en une abside de $7,90 \mathrm{~m}$ sur 5.15 $\mathrm{m}^{39}$. Là encore, l'hypocauste et le hérisson avaient complètement disparu ; les murs étaient entièrement récupérés. au s., a partir de l'angle n. du sondage QP 10, mais la tranchée de récupération apparaissait clairement, et le bourrelet de mortier laissé sur le radier de craie au pied de la face externe du mur. a permis de le localiser avec précision (fig. (i). Au s. du sondage QR 11, le rarlier est plaqué contre la fondation du mur d'enceinte (ou vice versa). Cela ne veut pas dire qu'ils ne sont pas contemporains, mais simplement que la construction s'est faite en deux étapes. On a pu observer d'autre part que la fondation du mur d'enceinte descendait en pente doure. devenant de moins en moins épaisse de l'o. vers l'e., jusqu'i se réduire a une mince couche de craie devant le radier des bains, face a l'absides. du caldarium. Cette fondation aurait-elle été entamée par les récupérateurs du mur s. du caldariam? Ou bien, hypothise plus vraisemblable, si la grosse fondation en "l " au s. des thermes est bien celle l'un praefurnium, les constructeurs n'ont-ils pas volontairement abaissé cette fondation. qui n'avail a supporter que la bouche du praefurnium vers le caldarium? si l'on ajoute it ces observations le fait qu'un praefurnium supplément aire a été construit contre l'abside du caldarium (sondage T. 12). on est tenté de conclure que celte abside, la partie la plus au s. et la plus chauffée des bains, est un laconicum.

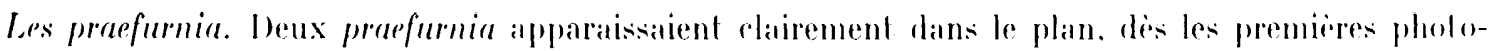
graphes aériennes. l'un. plus petil. al l'o. du tepidarium. el un autre. plus grand. il l'o. du caldarium. Les deux autres, moins bien conservés et d'interprétation plus dillicile. silues an s. el a l'e. du laconicum. romme on l'a vu plus haul. ont été révélés par les fouilles.

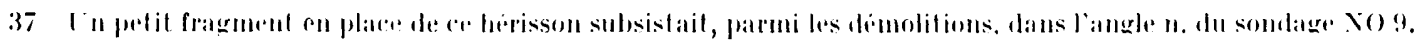

3x lin pen moins de a) p.

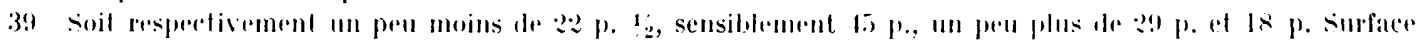
rhauffere: : "nviron 135 $\mathrm{m}^{2}$. 
Les vestiges du praefurnium du lepidarium (sondages $X 8$ et NO 9 ) étaient assez bien conservés et aisément intelligibles, la structure n'ayant pas subi de remaniement avant d'être détruite (peutêtre une reconstruction, mais sans modification du plan). Accolé au mur o. du tepidarium, le praefurnium est sensiblement carré40 (fig. 7) : il est délimité par un mur large de $0,70 \mathrm{~m}$, dont il restait jusqu'a 7 assises de mocllons, côté s. Il se divisc en deux parties : a l'e., posé sur un radier de craie lié au radier général des bains, et surmonté comme lui d'un hérisson de gros blocs, on trouve le foyer, dont le pavage de briques subsistait en grande partie ; le foyer est profond d'un peu plus de $0,80 \mathrm{~m}$, et se prolonge a travers le mur du tepidarium par un canal large de $1 \mathrm{~m}$. La partie o. du praefurnium, sur $1,50 \mathrm{~m}$ restant, ne comportait pas de fondation, en dehors de celles du mur. On y a retrouvé, posée sur l'argile. une série de couches de cendres : 4 couches bien distinctes de cendres noires, rougres ou blanches, dont l'épaisseur variait de quelques centimitres à une quinzaine de centimètres. Il faut voir lá une sorte de cendrier, plusieurs fois nettoyé, sans doute silué sous une grille sur laquelle brûlait le combustible. L'ensemble était trop arasé pour qu'on puisse se faire une idée de l'élévation (aspect de la paroi du canal, présence éventuelle de chaudières, etc.).

Le praefurnium latéral du caldarium apparaissait bien dans les photographies aériennes; il a un plan rectangulaire ${ }^{41}$; son mur $\mathrm{n}$. est large de $0.90 \mathrm{~m}$; les murs o. et s. n'ont que $0.85 \mathrm{~m}$; le mur s., dans le prolongement du prafurnium, se poursuit jusqu'au mur d'enceinte des thermes, isolant ainsi la cour des deux praefurnia de la cour située au s.e. Les sondagres P 8 et P 9 ont permis de préciser lis structure de ce praefurnium. légèrement différent du précédent, et de montrer qu'il arait subi un remaniement (fig. 8). Comme l'autre, il se divise en deux parties : il possède un cendrier a l'o., et à l'e., contre le caldarium, il repose sur un radier de craie tassée, comparable à celui du bàtiment des bains, mais non lié à lui, puiscu'il en est séparé par un espace d'environ $10 \mathrm{~cm}$ d'argile vierge. Ge radier a sensiblement $2,30 \mathrm{~m}$ d'extension vers l'o., mais sa limile o. n'est pas réguliere ; liée a ce radier, la fondalion de craie tassée du mur qui entoure le cendrier, en est contemporaine. Cette fondation n'est guère soignée : son orientation est décalée par rapport à celle du mur ; le haut de cette fondation n'est pas horizontal. Le mur, dont il reste 5 et 6 assises de petits pastoureaux, est renforcé sur la face o. par un rang de briques de chainage. Toujours còté o., l'assise inférieure du parement du mur s'épaissit vers le s., pour rattraper la pente suivie par la fondation. On se demande si ce praefurnium, assez bàclé dans son exécution, si on le compare au petit praefurnium du tepidarium, était prévu dans le plan initial des bains, et s'il n’a pas été rajouté pendant la construction de l'édifice : ainsi s'expliqueraient les contradictions entre les irrégularités observées, et. cependant. la parfaite insertion finale de ce praefurnium dans le plan d'ensemble.

Ce praefurnium, d'autre part, a été remanié : à l'o., on trouve, directement posés sur l'argile, des niveaux de cendres, comme dans le praefurnium du lepidarium. I l'e., sur le radier de craie, aurait dû se retrouver le foyer avec son parage de briques : on a rencontré, posé sur un hérisson de gros blocs de craie liés à l'argile, le canal, large d'environ $0.50 \mathrm{~m}$. qui traverse le mur du caldarium au-dessus de sa $4^{\mathrm{e}}$ assise de moellons, et, curieusement, se prolonge vers l'o. jusqu'au bout du hérisson, où il s'élarquil d'une quinzaine de centimètres. Le canal n'est pas pavé de briques, mais simplement enduit de mortier. sur lequel reposaient directement cendres et suie. Enfin, le parement intérieur du prafurnium (mur s.. seul fouillé) était refait, au-dessus du radier, en appareil irrégulier (fig. 9).

On est donc en présence de deux états : dans un premier temps. le foyer devait se situer, comme c'est normal, le long du mur du caldarium. Peut-être parce que le praefurnium chauffait trop. peutêtre parce qu'il était d'utilisation mal commode, peut-être à la suite d'usure ou d'accidents, on remania le praefurnium (second état, tel que nous l'avons retrouvé) en repoussiant vers l'o. l'emplacement du foyer, ce qui amenait à le réduire de moitié. Dans l'angle s.-o. du praefurnium, une fosse circulaire (diam. : $1,10 \mathrm{~m}$ ) trouait le hérisson : était-elle destinée au logement d'une chaudière?

$102 \mathrm{~m}$ sur $2,40 \mathrm{~m}$, mesures inlerieures.

$413,75 \mathrm{~m}$ sur $5,10 \mathrm{~m}$, mesures intíricures. 


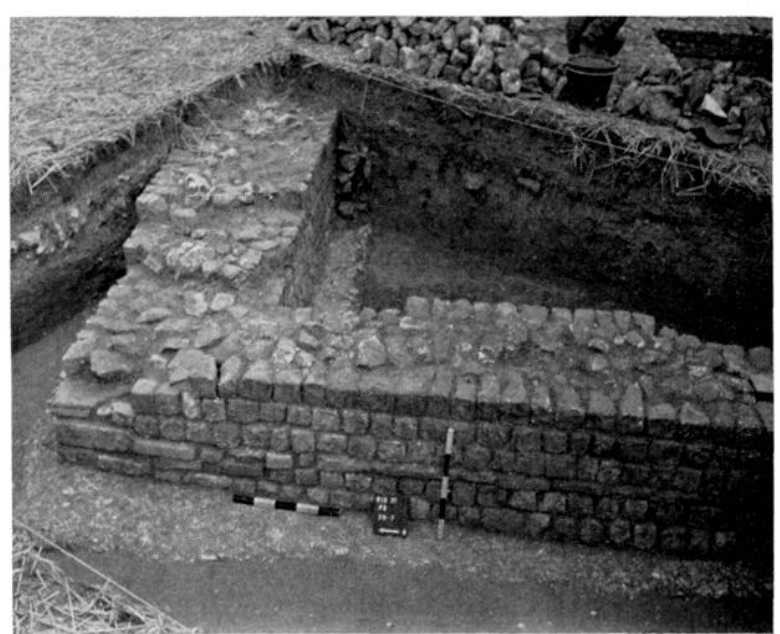

× L'angle ouest du praefurnium lalcral du caldarium. A gauche, le mur nord est un peu plus large. Al premier plan, le mur ouest rattrape la pente de la fondation. Il posside une assise de briques de chainage.

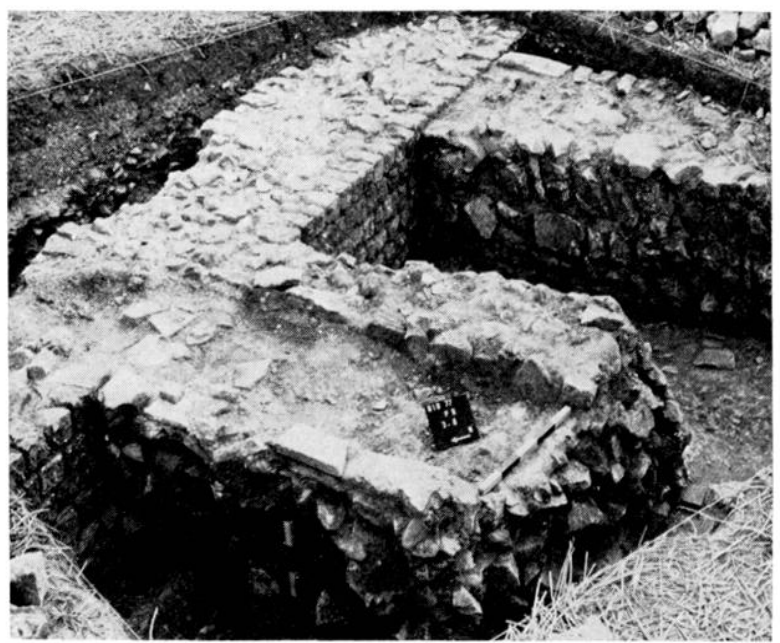

9 I.e foyer du praefurnium lateral du caldarium en cours de fouilles. 1 gauche, le mur du caldarium. Alu centre, partiellement fouilles, le canal du deuxieme etat, avec, en dessous, une partic du blocage. En haul, le mur sud du praefurnium, réparé en opus incertum.

Ce serait alors un remaniement du systeme d'implantation des chaudieres qui aurait conduit a modifier le praefurnium (?) $)^{42}$.

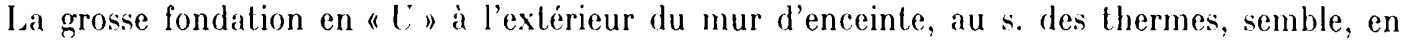
dernière analyse, avoir été celle d'un grand praefurnium, le plus grand des quatre; il en reste bien peu de choses (sondages TO 13, TP 12, TP 13, et QR 11) : une fondation de craie large de $2 \mathrm{~m}$, épaisse d'environ $0,50 \mathrm{~m}$, au plan légèrement trapézoïdal, bordée au s. par un petit fossé. A l'intérieur, entre la fondation et le mur d'enceinte des thermes, se trouvaient des sols de craie tassée recouverts de mortier beige, avec par endroits des cendres. Nous avons d'abord pensé, par comparaison avec le temple, à la fondation d'une colonnade, qui aurait constitué, còté rivière, une entrée monumentale des thermes, dans l'alignement du caldarium pris dans sa plus grande largeur : mais la faible épaisseur de la fondation exclut celte hypothese. Peut-il s'agir alors d'un nymphée, ou d'une piscine en plein air? L'absence de vestiges de décor architectonique, l'étroilesse de la structure (elle déborde le mur d'enceinte de moins de 5 m) iraient contre cette hypothèse. A saint-Bertrandde-Comminges, a Champlieu, etc., l'existence d'un praefurnium axial est certaine : c'est vraisemblablement dans le même sens qu'il faut interpréter notre fondation en " $l$ " " de Ribemont. A l'appui de cette hypothèse, on remarque que la tranchée de récupération du mur du laconicum (sondage QP 10) a été remblayée, au-dessus d'un premier niveau de mortier avec démolitions, par un niveau homogrène de cendres dont l'épaisseur dépasse par endroits $0,50 \mathrm{~m}$. Il est logique de faire venir cette cendre de notre praefurnium s., silué à $4 \mathrm{~m}$ de là. De mème. le remplissage de la tranchée de récupération du mur du laconicum et du mur d'enceinte (sondage QR 11) a donné beaucoup de charbon de bois. I'raefurnium done, mais on ne peut guère en dire plus.

Nous interpréterons aussi comme un praefurnium les vestiges de la petite construction adossée a l'angle e. du caldarium et du laconicum (sondage T 12 ) : un muret dans le prolongement du mur e. du caldarium, un autre petit mur (récupéré) perpendiculaire au premier, le tout posé sur un lit de craie carré de $3 \mathrm{~m}$ sur $3 \mathrm{~m}$, arec à l'intérieur, directement sur le morlier jaune qui surmonte la

42 Vitrove, le Archit., b, 10, recommande de placer les chaudieres all-dessus du foyer (supra hypocausim). Dans notre hypothese, une chaudiere aurait ete placée à coté du fover et du canal d'echappement des gaz. 


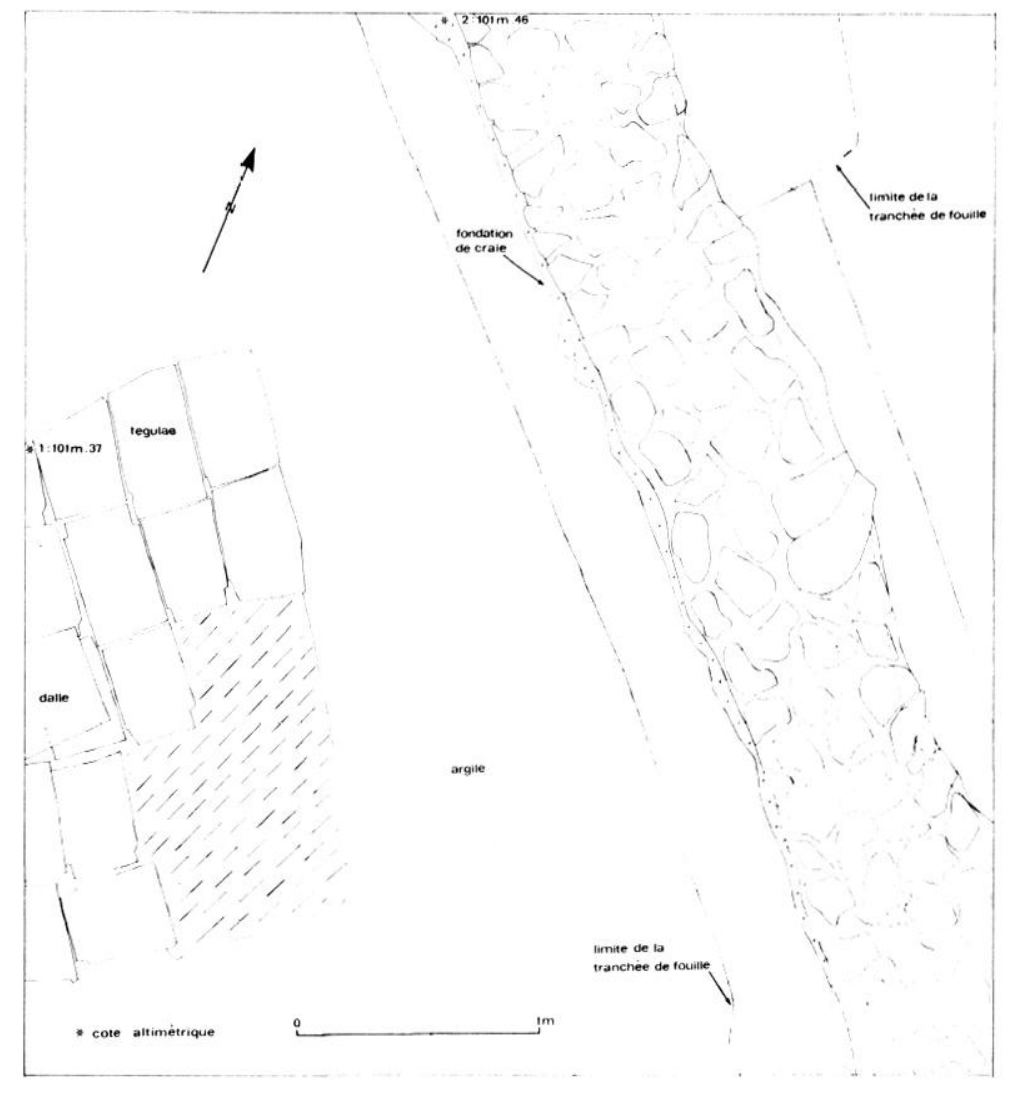

10 Le réservoir, dans la cour, à l'est du caldarium. A gauche, le fond du réservoir, partiellement fouillé. Les tegulae manquantes ont pait l'objet d'une récupération antique. $A$ droile, le mur d'enceinte.

craie, un épais niveau de cendres, scellé sous un épandage de tuiles brisées. Peu soigné et mal intégré dans le plan d'ensemble, ce praefurnium semble bien avoir été ajouté après coup (peut-être pour chauffer un bassin?)

Les annexes de service.

Il subsiste bien peu de vestiges dans les cours de service. Les sondages TM 13 et TN 13, à l'e. du caldarium ont été entièrement négatifs ; on a trouvé, immédiatement sous la terre arable, l'argile brune, sans structure construite, sans niveau de sol apparent ; le sol de la cour était-il en terre battue, ou a-t-il disparu du fait de l'arasement?

A l'o. du tepidarium, dans le sondage MN 8, une fondation de craie peu épaisse $(0,27 \mathrm{~m})$, avec un plan en " $Z$ 》, partiellement recouverte par les vestiges d'un médiocre blocage, reste impossible à interpréter : elle est postérieure au radier des bains, sur le bord duquel elle vient s'appuyer, mais elle passe sous l'angle n.-o. du mur du praefurnium du tepidarium, dont le cendrier a donc été réparé postérieurement à la construction de cette fondation. On se demande ce que pouvait être cet édifice, postérieur à la construction initiale des thermes, mais contemporain d'au moins une partie de leur période d'utilisation ... et qui curieusement se situe contre l'une des entrées possibles du bâtiment des bains. On peut conclure du moins que malgré la simplicité de leur plan, les thermes ont subi des remaniements de détail assez complexes.

A l'e. du caldarium, une petite tache carrée visible sur les photos aériennes nous a conduits à ouvrir un sondage (TL 13). Ce que nous avons trouvé, à un peu plus de $1 \mathrm{~m}$ derrière le mur d'enceinte, est un dallage de tegulae, posées directement sur l'argile; malgré une lacune (récupération des legulae dans l'angle s.-e.), et bien que nous n'ayons pas tout fouillé (un peu plus de la moitié de la 


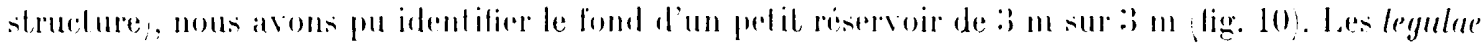
itaient poseces cote a cote bien i plat a un niveau qui se houvail 10 com plus profond que le haul de la fondation du mur d'enceinte voisin. Elles étaient libes a la chaux et fortement entartrées. Le remblai était composé de cendres et de fragments de tuiles; les borrls de la fosse, au-dessus du parage de legulae, etaient restes bien verticaux : on est donc en précense d un bassin, ou plus exactement diun réservoir, semi-enterré, dont les parois (colfrage de bois ou dalles de pierre? j ont été rérupérées au moment du démontage des thermes; après quoi, comme dans les fosses de réerupération des murs au s. des thermes, les démolisseurs ont remblayé avec des cendres. Aver la mare dont nous allons parler maintenant, re modeste réservoir est le seul vestige reconnu des adduclions d'eau dans les thermes.

I l'extérieur des thermes, au s.-e. de la palestre, les pholographies aériennes ${ }^{43}$ révélaient la présence d'une structure : une lache chare due à un épandage de démolitions. la fouille sondages I 11 a 13 , T' $i$ et TII 1:) a mont ré qu'il s'agissait d'une mare sub-circulaire d'un diamretre d'environ $10 \mathrm{~m}$, profonde de $4 \mathrm{~m}$ au centre dans une partie surcreusée, de $2 \mathrm{~m}$ dans son état initial ; le remblai élail, à l'o., scellé sous l'éboulis du parement du mur d'enceinte des thermes; d'où l'anomalie visible en prospection arienne. I e fond de la mare était constitué par un niveau d argile sableuse verdatre, eparis en moyenne de 0 , (6) m. riche en tessons quelques tessons du ier s. au contact de l'argile vierge, surtout des lessons de la deuxiome moitié du I $^{\mathrm{e}}$ s., dont une belle tète de lion. Inrag. to précoce.

Le plus intéressant était le remblai de la mare : la fouille a montré qu'on y avait déversé, all moment du démontage des thermes, lout ce qui ne pouvail pas ètre récupéré enduits peints, plaques de mortier de sol, conduits de fumée. Le remblaiement avait élé lait en commençant par le s. de la mare, où dominent les enduits peints, Landis qu'au n. les lubuli dominent. Pour le reste, le remblai était constitué d'un empilement de niveaux de mortier rose pulvérulent, et de niveaux de cendres, avec gà et là des fragments de briques de pileltes, des plaques de pavage, des baguetles moulurées en calcaire fin, et quelques morceaux de verre a vilre. L'intérêt principal de cette mare est donc de nous avoir livré une grande partie du décor intérieur des thermes, tel qu'il était soit au moment d'une réfection approfondie. soit hypothese qui aurait nos preférences au moment du demontage systématique. . Nous reviendrons plus loin sur celle question, dans la partie consacrée a la chronologie. Pour tout a qui concerne les enduits peints, on se reportera a l'article de $M^{\text {me }}$ Claudine Allag, ci-dessous (p. 107).

Il faut insister sur le fait que la mare, dont la profondeur ne devait guere à l'origine dépasser les $2 \mathrm{~m}$, a ele surereusie en son centre, jusqu'a une profondeur de $1 \mathrm{~m}$, par un puisard (diam. : $2 \mathrm{~m}$ sur $1,50 \mathrm{~m}$ ) au fond duquel onl ete lrouves des lessons de poterie commune de la premiere moilie

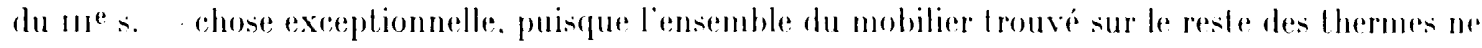
lépasie pas la lin du $\mathrm{u}^{\mathrm{e}} \mathrm{s}$.

Quelle était la fonction de cette mare? Elle a pu avoir une fonction primaire : arriere d'argile pour le lorehis d'un batiment anterieur aux thermes, ou, plus vraisemblablement. pour certaines parties des thermes qui avaient une éévation en lorchis comme le montre l'élude des enduits peints (voir infra); le volume d'argile reliré represente environ $100 \mathrm{~m}^{3}$. Eille a ensuite servi, soil romme réservoir d'eau, soit phutòt comme récephare pour les eaux usées : le depot d'argile verte, dans le fond, le montre rlairement. si elle a été surreusée, c'est sans doute pour la drainer, son bord o.. au pied mème du mur de la palestre risquant de menacer celui-ri.

Mabilement conceus selon un sehéma l res moderne pour son fomps. L destlermes pulaux

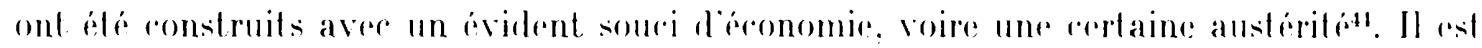

13) Inomalies de vigualation, voir supra fig. 9.

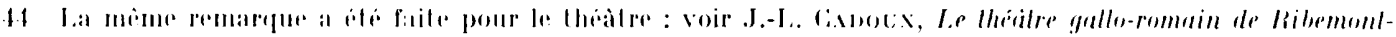

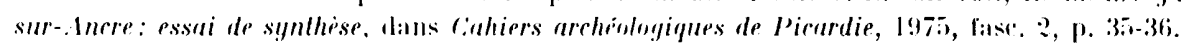


sûr que le bâtiment des bains n'était pas voùté, mais qu'il avait une couverture en charpente : le plan d'ensemble s'y prête bien, et surtout, il serait surprenant, s'il y avait eu des voùtes, de ne pas en retrouver la trace parmi les démolitions ${ }^{45}$. Le décor était modeste: des enduits peints d'une certaine banalité, comme on le verra dans l'article de Cl. Allag (infra, p. 107); pas de mosaïques de sol (aucune tesselle dans les démolitions), mais un lerrazzo assez rustique dans la pièce n. du tepidarium, et en d'autres endroits non déterminés, des plaques de calcaire fin blanc bordées de baguettes moulurées de la même matière; des murs en torchis dans certaines parties non précisables, puisque leur existence n'est connue que par l'examen des revers d'une série d'enduits peints. Beaucoup de rusticité aussi dans le système d'adduction d'eau, puisque nous n'avons trouvé aucune trace de conduit (n'y aurait-il pas eu des conduits en bois ?), et seulement un petit réservoir et une mare (le puits reste à trouver). Les thermes, comme le théâtre, faisaient, par leur sobriété, un singulier contraste avec le temple, pavé de marbres multicolores et couvert d'un riche décor sculpté et peint.

\section{Cihronologie}

Comme il est de riggle dans les édifices monumentaux, les thermes ont livré peu de mobilier datable - et cela d'autant plus qu'ils avaient subi un arasement systématique et profond. L'essentiel du mobilier est constitué d'apports extérieurs dans les remblais : c'est ainsi que, chose paradoxale dans des thermes, nous n'avons trouvé que à épingles en os. La chronologie repose donc au premier chef sur les lerminus donnés par les niveaux successifs de remblais ; nous avons toutefois eu la chance de trouver, dans la courette (?) à l'e. du frigidarium (sondage TI 10), ce qui semble bien être un dépôt de fondation, constitué de 2 petites céramiques intactes et de 2 monnaies, datables du règne de Trajan. Quelques tessons significatifs scellés au fond de la mare permettent de leur côlé, de préciser la date de la destruction des thermes.

A partir donc de bases médiocres mais cohérentes, nous avons établi une chronologie dont les grandes lignes sont les suivantes : a) le site est occupé avant la construction des thermes dès l'époque d'Auguste et pendant la période julio-claudienne jusqu'aux Flaviens. Aucune structure n'a été repérée, mais l'abondance relative du mobilier, surtout céramique, fait penser à un habitat; $b$ ) les thermes sont construits dans les premières années du ${ }_{\mathrm{II}}{ }^{\mathrm{e}}$ s. ; c) ils sont remaniés (piquetage des enduits peints et mise en place de nouvelles peintures; transformation du praefurnium à l'o. du caldarium; réparations au praefurnium du lepidarium) soit en une seule campagne à partir du règne de Marc-Aurèle, soit en plusieurs étapes dont une seule est datable ; $d$ ) la destruction systématique, dont l'un des aspects est le remblaiement de la mare, se placerait soit dès la fin du ${ }_{1}{ }^{\mathrm{e}}$ s., si l'on ne tenait compte que du mobilier recueilli dans les thermes, soit plutôt entre 210 et 260, comme l'indique l'examen du remblaiement de la mare; e) il est certain que le site est arasé et inoccupé depuis au moins les années 260 ap. J.-Ci.

45 Voir par exemple l'importance des blocs de la voùte écroulée dans les ruines du caldarium des thermes de Ciuny à Paris : P.-.I. Dcval, Paris Antique, Paris, 1961, p. 157. 
La première occupation est démontrée par la présence d'un mobilier gallo-romain précoce assez abondant mais qui a éte largement brassé, et qu'on retrouve dans des remblais argileux, à un peu tous les niveaux. mèlé à du mobilier plus récent. Deux monnaies au moins sur les douze retrouvées, un as à l'autel de Lyon, et un as de Néron, se rattachent a cotte première occupation, ainsi que deux fibules sur les trois retrouvées (voir l'inventaire un Annexe); d'une maniere significative, l'une des fibules, d'un type que l'on ne rencontre plus guere après le milieu du I ${ }^{\mathrm{er}}$ s., a été mise au jour dans le mème contexte qu'une fibule émaillée qui ne saurait être antérieure au $\mathrm{II}^{\mathrm{e}} \mathrm{s}$. La plupart des sondages ont livré des tessons de tradition de La Téne, des déchets de taille de silex et des lames ${ }^{46}$, quelques tessons de Lerra nigra et de lerra rubra, dont une signature ATITIM ou ATITIA, antérieure à 60 ap. J.-(C. ${ }^{77}$. Il est impossible de dire si les ossements animaux, huitres, cte, se rattachent à cette première occupation, ou aux occupations postérieures : du moins la prédominance de la céramique et son abondance permettent-elles de conclure à une occupation à caractère domestique. Aucun restige immeuble n’a été repéré : toute trace a certainement été détruite par la construction du bitiment des bains, et, aux abords, nos sondages ont été trop limités en surface pour qu'on puisse repérer une structure, s'il en subsistait. On voit que l'occupation gallo-romaine précoce (des le règne de Tibère pour la construction du temple) se retrouve d'embée d'un bout à l'autre du site, à $800 \mathrm{~m}$ de distance ${ }^{18}$.

La construction des thermes se situe à coup sûr pendant ou peu après les années 104111 ; cette date est donnée par la convergence de trois types d'indices : l'examen des céramiques donne des datations qui couvrent tout le $\mathrm{II}^{\mathrm{e}}$ s.; la série monétaire va de Domitien à Lucius Verus (hormis, pour la céramique comme pour les monnaies, le mobilier rattaché à la première occupation du site); enfin, par chance, nous avons mis au jour ce qu'on ne peut guère interpréter que comme un dépôt de fondation : il nous a donné un lerminus post quem précis.

La céramique, en général sous la forme de tessons isolés dans les remblais, comporte assez peu de sigillée (quelques feuilles d'eau à la barbotine sur Drag. 36 ; surtout des tessons de Drag. 37). Lensemble est typique du II s., de son début à son extrème fin. La poterie commune est dominante avec principalement des vases à col tronconique et des bols à pâte gris bleuté, à décor lissé de bandes horizontales. C'est une céramique très fréquente à Ribemont et en général chez les Ambiens, les Atrébates et les Morins. Les types retrouvés sur les thermes ont leur apogée localement dans la seconde moitié du ${ }_{11}{ }^{\mathrm{e}}$ s. $^{49}$.

La répartition des monnaies est assez significative : sur 12 monnaies, dont deux se rattachent à l'occupation du $\mathrm{I}^{\mathrm{er}}$ s., et une reste illisible, soit 9 monnaies utiles, on distingue une monnaie de Vespasien dans un contexte douteux et 3 monnaies de Iomitien,

16 Frequenls aussi dans les niveaux gallo-romains precoces foublés aux abords du temple.

17 Le meme nom de potier est connu a Blicquy Belgrique et a Camulodunnm (Angrleterre).

Ix ()n a retrouve de meme sur li sile du theitre, dans des niveaux également brasses, les traces d'une occupation comparable.

49 Vojr I). Vinnuksaa, La polerie commune gallo-romaine de Ribemont-sutr-Ancre, Memoire de Maîtrise, 1978, Ciniversite de Picardie, en partieulier p. 42 et pl. XXI et s. Voir D. BAYAR, La commercialisation de la céramique commune à Lmiens du milien du II"s. al lu fin du IIIes., dans Cahiers archiologiques de Picardie, n" 7, 1980, p. 1.17-209, lyores $25 b$ et $16,17,1 \times$. 


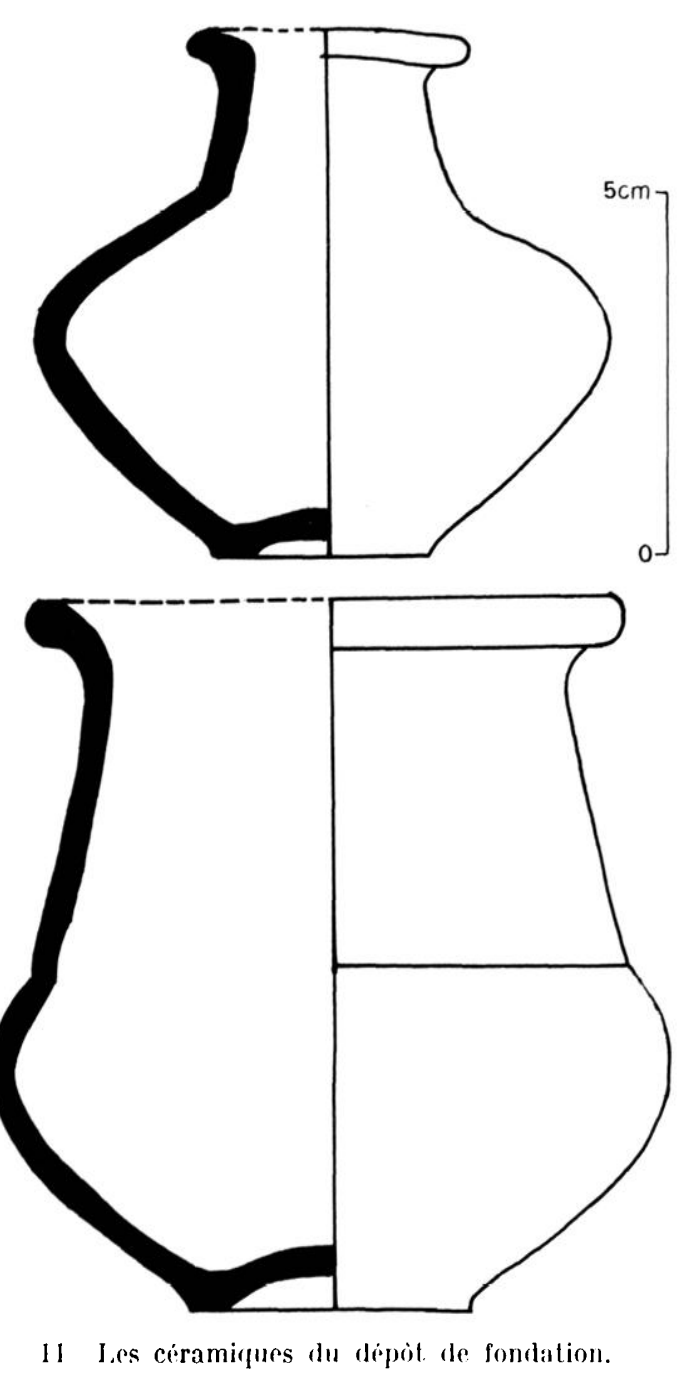

qui constituent avec les 2 monnaies du dépôt de fondation (Nerva et Trajan), et encore une monnaie de Trajan, un ensemble cohérent de 6 ou 7 monnaies des années 80 à environ 117 ap. J.-C. Le début du $\mathrm{II}^{\mathrm{e}}$ s. est donc clairement sur-représenté. Avec Hadrien et Lucius Verus, la fin de la série ne dépasse pas le ${ }_{11}{ }^{\mathrm{e}} \mathrm{s}$, et l'on notera en particulier l'absence totale à la fois du monnayage de Gallien et Postume, et de ces piécettes de bronze, imitations de Tétricus, qui circulent dans la deuxième moitié du $\operatorname{mir}^{\mathrm{e}}$ s. et pullulent partout ailleurs sur le sanctuaire de Ribemont.

La présomption que les thermes remonteraient au début du II s. a été transformée en quasi certitude par la découverte du dépòt de fondation. Dans le sondage TI 10 , a $1 \mathrm{~m}$ au s. du mur de la palestre et env. $1,50 \mathrm{~m}$ à l'e. de celui du frigidarium, on a rencontré successivement un épandage: d'argile contenant du mobilier de la première occupation (vers - $-0.80 \mathrm{~m}$ ), puis les traces d'un foyer, avec quelques ossements animaux, puis, groupés plus profondément dans l'argile vierge, deux monnaies à l'état neuf (à $-0,90 \mathrm{~m})$, un denier de Nerva daté de 97 ap. J.-Ci., un Dupondius de Trajan, entre 104 et 111 ap. .J.-C., enfin

deux petites poteries intactes en terre noire $(\dot{a}-1 \mathrm{~m})$, un gobelet à col tronconique posé couché sur le còté, et un flacon à panse globulaire, posé verticalement contre le précédent (fig. 11). Un gobelet à col tronconique semblable à celui de Ribemont a été trouvé à Amiens dans un contexte de la fin du $\mathrm{e}^{\mathrm{er}} \mathrm{s}^{50}$, et il s'agit en général d'un type de vase bien connu dans la région ${ }^{51}$.

L'interprétation de ect ensemble pose plus de problemes que sa datation; aucune trace de fosse n'est apparue au décapage: les poteries, comme les deux monnaies, qui elles aussi se touchaient, ont été placées dans un trou aussitòt rebouché avec la même argile

50) A la Gare routiere, dans le puils no 35, qui comporlait ped de mobilier deux vases tonmelels plus precoces'. mais pourrat bien avoir ele rebouche apres l'un des incendies de la ville sous lonitien. Fncore inedit, mais voir sur l'ensemble de cette foulle, T. Bkx RED.sB, Déconverle d'un noulean quartier d'Amiens romain à la fiare rouliere, dans Cahiers archéologiques de Picardie, 1978, p. 177 at s. In vase semblable mais incomplet est signalo, ibid., planche VIIJ, no 19 , dans unc fosse flavienne.

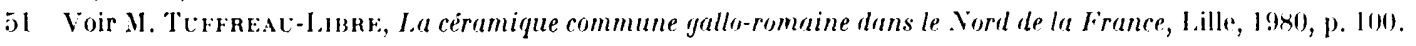


jaune que lon troure, sans molilier, au meme niveau. les poteries ne semblent avoir eu aucun contenu. Y avait-il une relation avec le foyer trouvé plus haut, à la base du niveau de remblaiement? Toutefois, mime si tout n'est pas facile à interpréter, on voit mal ce que pourrait ètre ce dépôt de deux monnaies neuves (chose exceptionnelle sur le site) et de deux vases intacts en position significative, si ce n'est un dépôt de fondation. Enterré sous le sol de la courette, trop tardif pour ctre lié à la première occupation du site, il ne peut être attribué quaux constructeurs des thermes. Nous n'hésiterons donc pas à lui attacher la plus grande importance et a placer la construction des thermes pendant ou peu après les années 10.1-111, date donnée par la monnaie de Trajan, élément le plus récent de l'ensemble.

L'utilisation des thermes pendant le $\mathrm{II}^{\mathrm{e}}$ s. est indirectement démontrée, on l'a va, par lanalyse du mobilier céramique épandu dans les niveaux de remblai ; un indice directeur confirme que les thermes fonctionnent encore sous, ou après, le règne de Narc-Aurèle : un as de Lucius Verus, lorùlé, a été trouvé dans la cendre du praefurnium ilu caldarium (sondage P 8). Ciest une monnaie avec le revers Forluna Redux, dont plusieurs séries ont été émises de $16 ; 3$ a 168 .

Il est possible que les réparations observées en divers points des thermes, et dont on a vu le détail dans la partie descriptive a arljonction d'un praefurnium d'appoint au s.-e. du caldarium, modification du praefurnium o. du caldarium, réparation au praefurnium du lepidarium) se soient faites, non pas au coup par coup, mais a l'occasion d'une seule campagne, qui aurait eu lieu au plus tòt sous le règne de Marc-Aurèle. Les principaux indices sont donnés par te mobilier trouvé au démontage du second etat du praefurnium o. du caldarium dans le sondage P 9. A l'intérieur du blocage construit à l'emplacement du foyer du premier etat, la découverte d'une lève de mortier Drag. 45 montre que la réparation ne saurait ètre antérieure à Marc-Aurèle. On a noté la présence d'assez nombreux fragments d'enduits peints, entre le blocage et la fondation primitive du foyer : ces enduits peints sont assez différents de ceux, avee deux couches de peinture, trouvés dans la mare. On pourrait y voir les vestiges d'un premier état du decor intérieur des thermes : les enduits peints de la mare appartiendraient alors à un serond état postérieur au milieu du $\mathrm{II}^{\mathrm{e}}$ s. Mais ces quelques enduits peints trowes dans le praefurnium peurent aussi bien venir de n'importe quel mur, intérieur ou extérieur, sans que cela implique une réfection globale des enduits intéricurs.

Ene chose est nette : depuis 'Trajan jusqu'au moins à la fin du ${ }^{2}{ }^{\mathrm{e}}$ s., les thermes ont été utilisés et largement utilisés, à en juger par l'épaisseur de la suie -- encore comme fraiche - trouvéc à l'intérieur des lubuli jetés dans la mare, et par l'abondance des cendres répandues autour des vestiges des prafurnia. Lne autre chose est également nette: même si toutes les réparations exécutées dans les thermes ne l'ont pas èté en mème temps, une campagne de restaurations, sous ou peu après Marc-Aurèle, est attestée : cela rejoint les observations faites sur le théatre (transformation en amphithéatre-théatre après 160) et le temple (réalisation du dernier décor sculpté à partir des règnes d'Antonin ou Marc-Aurèle).

Il reste la question de la démolition des thermes. De nombreux indices montrent qu'elle a été systématique el ordonnée. Les murs ont été arasés très proprement au niveau de la base des hypocaustes, dont les briques de pilettes ont toutes été soigneusement récupérées. Partout dans les sonrlagess, le volume des démolitions était très faible : presque 


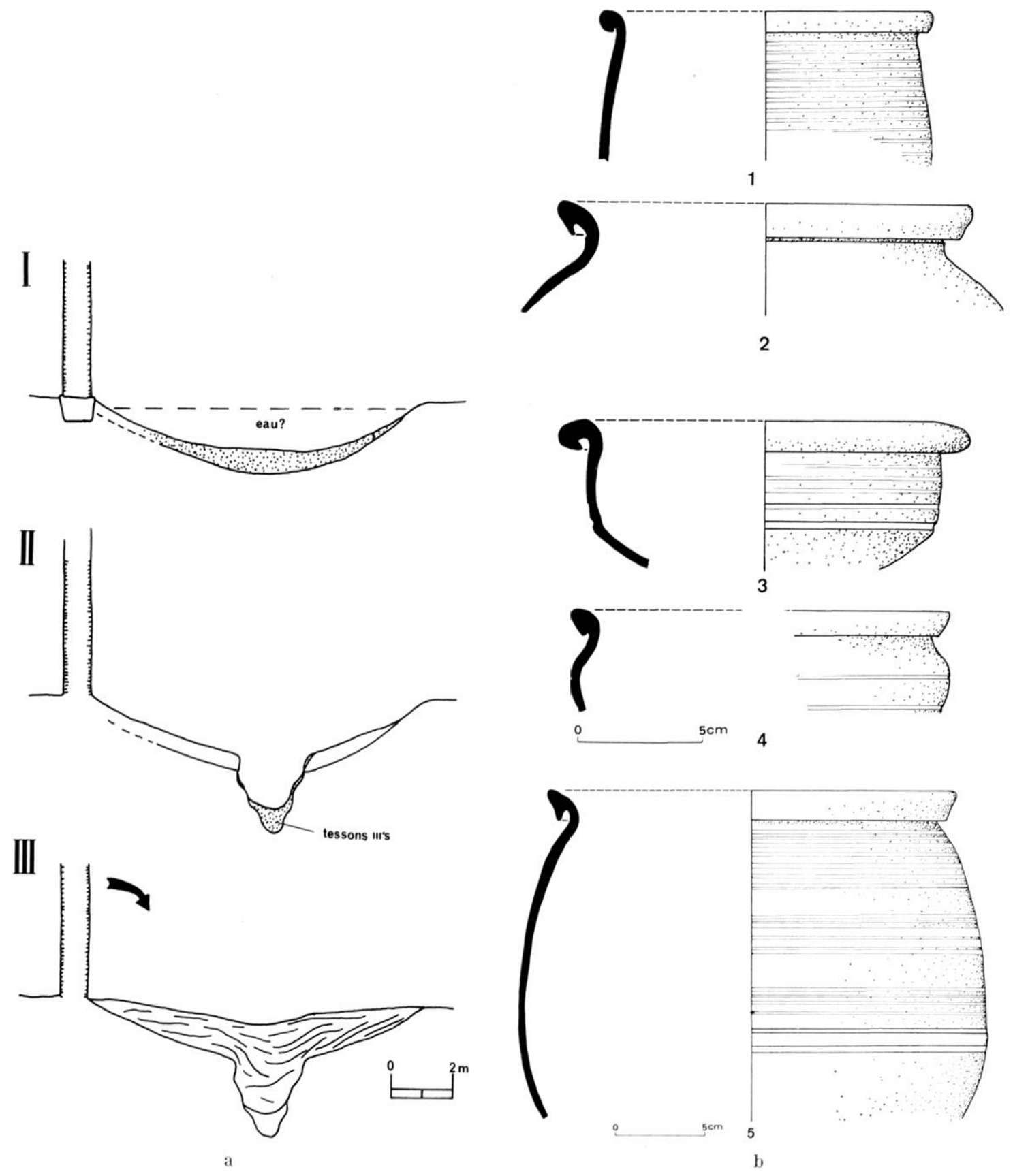

12 Ia mare et le lerminus post quem de la destruction des thermes. a, Schema de l'utilisation et du rebouchage de la mare. b, ciéramiques de la premiere moitié du II $^{\mathrm{e}} \mathrm{s}$. trouvées au fond du puisard central.

tout avait été enlevé, emporté. On a vu, dans la description du remblai de la mare, supra, comment les démolisseurs, venant des bains au s. de la mare, avaient d'abord jeté les enduits peints et du mortier en blocs brisés, puis, en avancant, les conduits de fumée, avant de s'attaquer aux moellons de parement, qui, eux avaient été emportés.

On a pu faire aussi d'intéressantes observations dans le coin n.-o. de la pièce (apodyterium?) au n. du lepidarium (sondage XI 9) : comme souvent les démolisseurs s’étaient 
intéressés particulièrement aux moellons contenus dans l'angle de la pièce (on observe la même chose dans l’angle n.-o. du frigidarium, sondage TI 8); ils avaient brisé le sol de la pièce pour aller chercher les moellons de parement du mur n. et les avaient arrachés, laissant leur empreinte dans le blocage. Ils ne s’étaient pas embarrassés à jeter bien loin les blocs de sol : deux gros blocs ont été retrouvés tels qu'ils avaient été posés, à plat un mètre plus à l'e., sur la partie non détruite du sol. Or entre les blocs enlevés et le sol en place ne se trouvait qu'une très fine couche de cendre, et aucune autre salissure - - une cendre qui peut bien provenir du praefurnium voisin ou de l'hypocauste qu'on était en train de démolir en mème temps; ce vestige de l'instant même de la démolition des thermes confirme bien notre impression générale : elle s'est faite d'une manière ordonnée, dans un laps de temps très court. Une destruction volontaire et bien organisée.

On aimerait évidemment parvenir à dater avec précision ce démontage. C'est une entreprise difficile: on sait d'expérience que si les éléments susceptibles de dater une construction arrivent toujours à être trouvés, ceux qui permettent de dater une démolition sont plus fugaces; d'autre part, la démolition des thermes se situe au in ${ }^{\mathrm{e}}$ s., période où les chronomètres se font plus rares et incertains. Nous sommes parvenus malgré tout à obtenir une fourchette : après 210 , avant 260 certainement, peut-être un peu plus tôt.

Voici les éléments sur lesquels nous nous sommes appuyés. Tout d'abord, le mobilier céramique comme la série monétaire ne semblent pas dépasser la fin du ${ }_{\mathrm{II}} \mathrm{e}$ s. Il est vrai qu'il s'agit de mobilier en position peu significative et que, dans la céramique au moins, des éléments plus tardifs ont pu nous échapper, faute d'être reconnus. On serait donc tenté, de prime abord, de situer la destruction des thermes peu après la fin du II $^{\mathrm{e}} \mathrm{s}$. D'autre part le lerminus ante quem est à situer dans les années 260, date à partir de laquelle les émissions contemporaines de l'Empire Caulois s'infiltrent partout : or, elles sont complètement absentes ici, de mème que tout mobilier postérieur.

En second lieu, l'examen particulièrement attentif de la mare et de son remblai (fig. 12) nous ont apporté une précision supplémentaire. On a vu, supra, que la mare avait été surcreusée en son centre, par une sorte de puisard, qui descendait jusqu'à $4 \mathrm{~m}$ de profondeur. Or, le mobilier trouvé dans la boue au fond du puisard est de première importance pour notre propos : on y rencontre, avec des éléments du ${ }_{\mathrm{II}}{ }^{\mathrm{e}} \mathrm{s}$. tardif (assiette dite "de Bavai ", imitation de lerra nigra, quelques tessons de céramique a couverte métallescente), plusicurs tessons assez gros et bien identifiables de céramiques que l'on parvient, depuis peu, à dater dans une fourchette de 210 à 250 ap. J.-C..52.

Cous avons donc là un terminus posl quem de la destruction des thermes, ces tessons étant scellés sous les vestiges du décor intérieur des bains, déversés dans la mare. Toutefois, un doute pouvait subsister, car la stratigraphie du centre de la mare, au-dessus du puisard, n'était pas parfaitement claire : l'éboulement du parement du mur e. de la palestre ne va pas jusque-là, et l'alternance des couches de mortier, cendres, enduits peints, etc., est beaucoup moins nette au centre de la mare que sur les bords. Bref, on aurait pu penser

52 Narmile et écuelles en terre noire el grise, semblables aux céramiques décrites par 1). Bayard, La commercialisalion de la céramique commune à Amiens, du milieu du $I^{\mathrm{e}} \mathrm{s}$. à la fin du IIJ $\mathrm{s}$., dans Cahiers Archéologiques de Picardie, 1980, p. 170 et s., nos II-15, IV-34, IV-93, V-6 et VIII-15. Figalement à Ribemont, 1). Verserascil, op. cil., pl. VI a VIII, dans un dipotoir, pres du temple, daté en particulier par une monnaie d'Alexandre Sévère. 
que le puisard central avait été creusé après le remblaiement de la mare, et que le lerminus post quem n’était donc pas valable. Plusieurs arguments font tomber l'objection : comment et pourquoi aurait-on pu, un certain temps après l'arasement du site, recreuser un puisard dans une mare remblayée, et qui mieux est en tombant exactement au milieu, alors quion ne devait plus guère la voir au sol ? Sur les bords du puisard, où l'on observe des paliers successifs, et au fond, se trouvait la mème argile verte que dans le fond de la mare, premier état. Il faut donc plutòt envisager le schéma suivant : la mare, à l'époque où elle c'st en usage, un usage en rapport avec le service des thermes, est surcreusece en son centre; peut-itre a-t-on besoin de la drainer, surtout si elle menace le mur e. de la palestre au pied duquel elle s'étale. Au moment de la démolition des thermes, pendant ou apres les années 210-250, la mare et son puisard sont comblés en une seule fois. Le parement extérieur du mur de la palestre n'est pas récupéré, peut-être parce que l'opération aurait été dangereuse ; il est basculé (ou bascule tout seul) sur la mare remblayée, ce qui a l'avantage de colmater l'ensemble avant la mise à niveau définitive. On ne doit done plus hésiter à retenir une date entre 210 et 250 comme lerminus post quem de la destruction, le lerminus anle quem étant, on l'a vu, fixé autour de 260), époque où les monnaies de Ciallien, Postume, puis les imitations de Tétricus recommencent à circuler, et bientìt à pulluler, mais sont absentes des thermes. Il est sûr qu'après cette date, à la différence du théatre loujours en activité au Ive s., de la "zone artisanale " et des abords du temple où l'orcupation dure jusqu'a la fin du $\mathrm{IV}^{\mathrm{e}} \mathrm{s}$, il n'y a plus rien a l'emplarement des thermes.

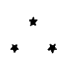

Malgré l'état d'arasement profond des thermes, bien que les surfaces fouillées aient été volontairement réduites, et que les fouilles se soient limités à deux campagnes d'été, on peut juger de l'importance des résultats obtenus : le plan a été précisé ; diverses structures qui n'apparaissaient pas ou n’étaient pas interprétables d'après les photos aériennes, ont été identifiées; des renseignements sur l'aspect des thermes en élévation, et leur décor. sur les méthodes de construction, ont été obtenus; mais le plus important est qu'une chronologie relativement précise de l'édifice peut maintenant être proposée. Ierniers venus de la série des monuments du sanctuaire, les thermes sont alandonnés les premiers; leur longévité aura à peine dépassé un siècle : on no peut pourtant pas dire quiils n'ont pas cu de succès, puisque tout concourt à démontrer qu'ils ont été intensivement utilisés. Pourquoi alors leur destruction volontaire? Ies thermes sont, de tous les monuments, ceux dont l'entretien est le plus coûteux, en personnel, en fournitures, en réparations. On regrette de ne pouvoir dater plus rigoureusement leur destruction : une date précise aurait peut-être permis de savoir si la fermeture de l'établissement découlait de difficultés conjoncturelles ou strurturelles.

Didlier BaYARl et Jean-Lonis (ianolx

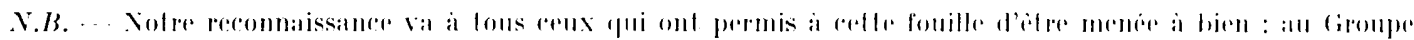

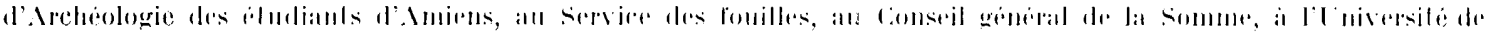

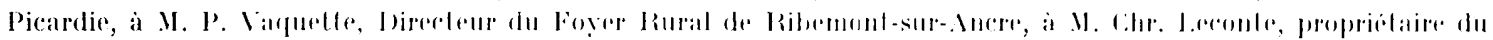

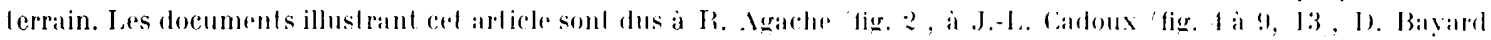

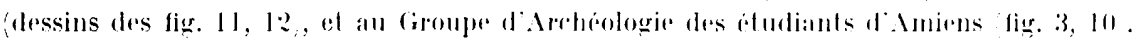




\section{ANEXE}

\section{INENTARE DHS MONAALS PAR ORDRE CHRONOLOGIQLE}

1 . Ls d'Auguste ou Tibère (revers à l'autel de lyon), très usé, trouvé dans un niveau d'argile remaniée, à $0,60 \mathrm{~m}$ sous le sol de la palestre, prís de la fondation, devant l'angle n.-e. du frigidarium (sondage TI 9).

2 As de Yéron (R.I.C., n ${ }^{0} 329$; isolé dans l'argile, sans stratigraphie, à $\left.0,6.5\right)$ m de profondeur, a $8 \mathrm{~m}$ au $\mathrm{nl}$. du mur extérieur $\mathrm{n}$. de la palestre (sondage négatif $\mathrm{E} 7$, non figuré sur le plan de la fig. 3 ).

3) Dupondius de Vespasien (Cohen, $\mathrm{n}^{0}$ 469, 71 ap. J.-C.), mème contexte que no $1,1 \mathrm{~m}$ plus à l'o.

4 Denier de Domitien (Cohen, $\mathrm{n}^{0} 554,80$ ap. J.-G.), trouve entre le réservoir et le mur extérieur des thermes, dans un niveau de terre au-dessus de l'argile vierge (sondage TL 13).

5. IIoyen bronze brùlé de Jomitien, peu lisible, trouvé dans un niveau de terre cendreuse remaniée, au-dessus de la fondation du grand praefurnium au s. du caldarium (sondage TP 12).

(5) As de I)omitien (R.I.C., no 394, 9()-91 ap. J.-C.), découvert contre l'angle n.-o. du mur du praefurnium du tepidarium, dans un remblai de craie granuleuse, à la base du niveau où s'observe une reprise dans le mur (sondage $\times 8$ ).

Dépót de fondalion.

7 Denier de Nerva (Cohen, $\mathrm{n}^{0}$ 6, C.R.K.B.M., $\mathrm{n}^{0} 52,97$ ap. J.-C.) à l'état neuf, découvert avec 2 poteries et la monnaie suivante, dans l'argile, a $0,90 \mathrm{~m}$ de profondeur, a $1 \mathrm{~m}$ au s. du mur séparant la courette à l'e. du frigidarium, de la palestre (sondage T I 10).

8 Dupondius de Trajan (Cohen, $\mathrm{n}^{0}$ 606, C.K.E.B.M.M. no 903, entre 104 et 111 ap. J.-C.), état neuf, mème contexte.

9 Dupondius de Trajan assez usé (Cohen, $\mathrm{n}^{0} 173$ ?), trouvé dans l'argile au bord de la mare, à $0,60 \mathrm{~m}$ devant le mur extérieur des thermes (sondage TII 12 .

10 Is d'IIadrien (Cohen, $n^{\circ} 298$ ), trouvé dans une terre sans stratigraphie et a 0,40 m de la surface, a $1.50 \mathrm{~m}$ a l'e. de la fondation du grand praefurnium s. (sondage TO 13).

11 Is de Lucius Verus très abìmé, brûlé (sans doute Gohen, nos 99, 104 ou 109, 1633 a 168 ap. J.-C.), trouve dans la cendre du praefurnium du caldarium (sondage P 8).

12 Monnaie illisible à flan mince, diam. $23 \mathrm{~mm}$, découverte dans la courette à l'e. du frigidarium, dans l'argile de remblai a moins de $1 \mathrm{~m}$ du dépôt de fondation (sondage TI 10).

\section{INVENTAIRF DES FIBULES}

1 Fibule à ressort nu, are filiforme brisé et porte-ardillon plein (cf. Lerat, Calalogue..., nos 34 à 36 ler), d'Auguste à la fin du ${ }^{\mathrm{e} r}$ s., découverte dans un remblai d'argile, postérieur à la démolition du portique, à l'e. de la palestre (sondage I 10).

2 Fibule à ressort protégé. are à cannelures, à raccord droit avec le couvre-ressort (cf. Lerat, ibid., $\left.\mathrm{n}^{\text {os }} 85-87\right), 50$ av.-50 ap. J.-C., dans un niveau d'argile remaniée asse\% profond $(0,90 \mathrm{~m})$ a $1 \mathrm{~m}$ au $\mathrm{n}$. de la fondation du praefurnium du tepidarium (sondage $\times 8$ ).

3 Fibule à charniere, ilamée, plarque reclangulaire a profil cambré, bordée d'un grènetis, avec au centre une bande champlevée avec cinq carrés portant des traces d'émail (cf. Lerat, ibid., nos $256 \mathrm{et} \mathrm{s}$.), ${ }^{\mathrm{e}} \mathrm{s}$. Mème contexte que la précédente, a $1 \mathrm{~m}$ plus au $n$. (sondage $X$ S).

\section{J.-L. Ciadotx}

